\title{
Limits on Simulation Approaches in Intuitive Physics
}

\author{
Ethan Ludwin-Peery, Neil Bramley, Ernest Davis, and Todd M. Gureckis
}

January 12, 2021

\begin{abstract}
A popular explanation of the human ability for physical reasoning is that it depends on a sophisticated ability to perform mental simulations. According to this perspective, physical reasoning problems are approached by repeatedly simulating relevant aspects of a scenario, with noise, and making judgments based on aggregation over these simulations. In this paper, we describe three core tenets of simulation approaches, theoretical commitments that must be present in order for a simulation approach to be viable. The identification of these tenets threatens the plausibility of simulation as a theory of physical reasoning, because they appear to be incompatible with what we know about cognition more generally. To investigate this apparent contradiction, we describe three experiments involving simple physical judgments and predictions, and argue their results challenge these core predictions of theories of mental simulation.
\end{abstract}

\section{Introduction}

Our ability to reason about physical and mechanical systems is an important and unique aspect of human intelligence. Much of our scientific and technical progress owes to our ability to reason about systems that do not yet exist and to then build them. Successful interaction with our environment often requires reasoning about the physical world; for example, we might need to predict if a stack of books on a desk is unstable, or judge when to safely enter a rotating door.

Several decades of research in cognitive science suggest that these abilities rely on some type of "mental model" of the scenario - a representation of the the objects with the system or situation and their interactions according to the rules of physics (P. Johnson-Laird, 1998; P. N. JohnsonLaird, 1980, 2010). However, the detailed nature of these mental models remains only partially understood, evidenced by the fact that we have yet to implement artificial intelligence systems which the same flexibility and performance as humans at physical reasoning tasks. These judgments are ubiquitous, but the mental processes that support this ability remain poorly understood.

If the way that people intuitively reason about physical system remains a mystery, it is not for a lack of proposals and attempts. Over the past several decades, researchers have explored heuristic approaches (Kozhevnikov \& Hegarty, 2001; Schwartz \& Black, 1996b), qualitative reasoning systems which encode and reason about qualitative relationships between variables (Forbus, 1984; Forbus \& Gentner, 1983; Klenk \& Forbus, 2009), abstract causal reasoning (Griffiths \& Tenenbaum, 2007; Holmes \& Wolff, 2010; Sloman, 2005; Wolff, 2007; Wolff \& Barbey, 2015), analogy (Gentner, 1983; Gentner \& Gentner, 1983), and pattern-matching approaches similar to perception (Barsalou et al., 1999; Firestone \& Scholl, 2016, 2017).

Perhaps the most influential approach is based on mental simulation. In a simulation, a program starts with an initial situation, and then applies the relevant dynamic laws of physics to compute what will happen over a series of very short time steps: in effect, computing a "movie" of the progress of the situation. In scientific and technical settings, simulation is a common technique used for physical reasoning, and is successful in applications ranging from modeling molecular interactions to designing realistic video games. ${ }^{1}$ Simulation based approaches to physical reasoning draw from a classic debate in cognitive psychology concerning the nature of mental imagery. Simulation theory is in some sense the heir of the "analog" representation theory which held that image-like mental representations were isomorphic in many important ways with the physical world (Hegarty, 2004b; Pylyshyn, 2002; Shepard \& Metzler, 1988).

\footnotetext{
${ }^{1}$ In the context of video games, the simulation program is often called a "physics engine".
} 
In recent years, simulation-based models of physical reasoning have become very popular (Davis \& Marcus, 2015, 2016; Hegarty, 2004a). In particular, a more specific, and concrete account of mental simulation has been proposed that can be instantiated as computer models (Battaglia, Hamrick, \& Tenenbaum, 2013; Dasgupta, Smith, Schulz, Tenenbaum, \& Gershman, 2018; Hamrick, Battaglia, Griffiths, \& Tenenbaum, 2016; Hamrick, Smith, Griffiths, \& Vul, 2015; Sanborn, 2014; Sanborn, Mansinghka, \& Griffiths, 2013; Smith, Peres, Vul, \& Tenebaum, 2017; Smith \& Tenenbaum, 2013; Ullman, Spelke, Battaglia, \& Tenenbaum, 2017). This approach to developing simulation models has a number of important strengths. First, the new simulation theories derive predictions using a generative model of the environment based on a video game physics engine. This gives the theory broad coverage in the sense that it can apply to many arbitrary physical situations, unlike for instance causal or analogical models which are often constructed by hand for small domains. In addition, simulation models correctly predict significant aspects of human reasoning about many different physical situations. These include judging whether a 3-D tower of blocks will collapse, determining the direction in which such a tower will fall (Battaglia et al., 2013), evaluating the extent to which a particular brick in a 2-D block tower is responsible for the rest of the bricks staying on a table (Gerstenberg, Zhou, Smith, \& Tenenbaum, 2017), predicting the destination of a virtual ball on a 2-D bumper table (Smith \& Tenenbaum, 2013), and predicting the proportion of a poured liquid that will end up on either side of a divider (Bates, Yildirim, Tenenbaum, \& Battaglia, 2015), among others.

Despite these advances, the plausibility of simulation as a cognitive theory has also been contested (cf., Davis \& Marcus, 2015; Marcus \& Davis, 2013). One of the major criticisms of this approach is the apparent incompatibility of accurate physical simulation with decades of psychological work documenting human errors in simplified physical reasoning tasks (Kubricht, Holyoak, \& Lu, 2017) such as predicting the trajectory of balls exiting curved tubes (McCloskey, Caramazza, \& Green, 1980), the tipping direction of balance beams (Siegler, 1976), and the behavior of dynamic mechanical systems such as wheels (Proffitt, Kaiser, \& Whelan, 1990) or pullies (Hegarty, 1992).

Other issues arise from computational considerations. For instance, if a closed can half full of sand is shaken, simulating its behaviour would require calculating the collisions of all the grains of sand and doing so many times to account for uncertainty about their positions. If you simply want to predict whether the sand will remain in the can, that can be done through the application of a simple containment heuristic (Davis \& Marcus, 2016; Davis, Marcus, \& Frazier-Logue, 2017; Smith, Dechter, Tenenbaum, \& Vul, 2013; Smith et al., 2017).

\subsection{Probabilistic Mental Simulation Theory}

A key feature of modern simulation-based approaches to physical reasoning is the connection and use of probabilistic models of cognition. Instead of committing to a single mental model, these accounts propose that multiple simulations are run from a range of different initial configurations. This aspect of these theories helps to capture the imprecise and qualitative nature of human physical reasoning.

For instance, imagine that a person is asked to predict whether a tower of wooden Jenga blocks will fall over. A probabilistic mental simulation begins by assuming that the reasoner has an imperfect perception of the positions of the blocks (i.e., their precise locations in physical space) and other physical properties, owing to perceptual limitations and occlusion. Based on this uncertain percept, the reasoner creates a number of slightly different towers. Each tower is altered according to random noise, to account for perceptual uncertainty. These simulations are in some cases further perturbed by noise applied to interim states (e.g. following collisions Smith \& Vul, 2013). A reasoner might start with ten such initial towers and run a (possibly noisy) physics simulation forward until some natural termination point determines the person's subjective impression. The reasoner can then approximate the probability of different outcomes, given their subjective uncertainty about the scene via Monte Carlo approximation; that is, by counting the frequency of different outcomes in the sample of simulations. For example, if 8 of the 10 simulated towers fall over then a reasoner might estimate an $80 \%$ probability that the structure is unstable (Battaglia et al., 2013). For the purposes of this paper, we refer to this approach as "probabilistic mental simulation theory" (PMST).

Ullman et al. (2017) propose a modification of PMST in which the mental "physics engine" that powers human reasoning uses some of the same shortcuts and approximations that current videogame engines do. Contemporary video game physics engines generate 3D physics environments 
which appear realistic and compelling. However, for reasons of computational efficiency, most video game engines use several "tricks" and shortcuts which deviate from a complete physical simulation. For instance, one simplification is to put objects "to slee" which essentially means to turn of physical tracking for an object when it is at rest and has not been part of a collision with another rigid body object in a certain amount of time. The hypothesis in the "video game engine in the head" theory is that the mind uses similar shortcut and in fact the tricks used in the video game industry might actually serve as inspirations for cognitive theories. This theory is a substantial refinement of the PMST approach, particularly because by assuming that there are computation short-cut that operate on processes and representations of the world, it introduces the possibility of systematic errors in reasoning that seem to manifest in many tasks and situations. However, at its core, the theory still relies on iterative simulation, aggregated across many samples in order to make prediction. In this sense, the "video game engine in the head" hypothesis is a precise and constrained version of the more general class of PMST.

The present paper describes a number of empirical tests of PMST. There is a broad consensus in the field that people use a variety of mental strategies in physical reasoning, depending on the situation and the task at hand; but there is no agreement on how much of physical reasoning a particular strategy like PMST accounts for, or under what circumstances people use one strategy or another. We begin in the next section by describing three core tenets of PMST that transcend specific applications of the theory but, rather, make important testable claims about human physical reasoning. We then describe a sequence of novel experiments that try to expose these principles by setting up pre-registered ${ }^{2}$ edge cases where we might expect the predictions made by PMST to fail.

We designed our experiments to use very similar designs and stimuli as tasks that have previously been used to support the PMST theory. Thus, they provide helpful data for establishing principled limiting cases on models of this type. The results of our experiments are hard to reconcile with PMST, and so we consider several alternative theories of how people perform each task. Modeling based off of our findings suggests that in many cases, behavior on these tasks is better explained by simple heuristics than by simulation, even when the tasks are very similar to past paradigms where behavior has been attributed to simulation.

More generally, this work lays the foundation for determining the limiting cases for the use of mental simulation in intuitive physics, and provides some information as to the strategies employed when simulation reaches its limits.

\subsection{Three key principles of probabilistic mental simulation theory (PMST)}

An agent using a probabilistic simulation of the physical world to solve physical reasoning problems should exhibit certain behavioral patterns. While it has been argued that mental simulation may include several limitations and shortcuts (Ullman et al., 2017), the principles outlined here are an attempt to find core tenets of the approach that are inevitable consequences of any simulationbased theory. Consistent violation of these patterns would suggest that human reasoning about physical scenes uses a different type of mental process. Specifically, PMST implies the following three principles, which we test in the three experiments.

\subsubsection{Principle 1: Persistence of simulated objects}

When making predictions about a physical scene, a reasoner using PMST is required to maintain at least some representation of all interacting objects within all simulations/samples. For instance, objects occupy particular locations within space and time, and a mental simulation must represent this position and update it according to the rules of physics. This is a core aspect of the theory, because dropping or losing track of objects destroys the viability of the simulation approach. Objects might be simplified or chunked together with other objects, but they need to be represented in some way throughout. "Deleting" an object from a mental simulation will radically alter possible outcomes and subsequent predictions (making it in fact a completely different simulation) due to chaotic and nonlinear interactions between objects.

For example, imagine a person looking at a table. If their mental simulation were to accidentally delete its representation of one of the table legs (even temporarily), that would result in a major disruption to the simulation (e.g., the otherwise static table may begin to dynamically

\footnotetext{
${ }^{2}$ Preregistration here.
} 
fall). Keeping track of the location of objects in space and time and accounting properly for their movements (and the consequences of their movements on other objects) is fundamental to what it means to "simulate" a physical scene: a simulation-based representation of a physical scene must be largely isomorphic to the real world in order to maintain coherent predictions about the future. Physics engines do make mistakes and approximations, but deleting or radically altering objects is not the kind of mistake they make.

There are, of course, some cases where objects may be ignored. A boundedly rational reasoner is not obliged to simulate every object in the universe to make an accurate local prediction. In making judgments about the behaviour of a tower of blocks on your kitchen table, you can safely ignore the position of objects located on Mars, or even in the next room, without reducing the accuracy of a simulation-based judgment. Events in a closed container, in a distant location, or on a small enough scale, can be and routinely are ignored (though how the mind manages to effectively draw these boundaries is itself a complex question).

Even within a "scene", there may be cases where some objects can be ignored. In video game physics engines, for example, objects at rest are often put to "sleep" to save on computation, and woken up when their support changes or a collision occurs. It it has been suggested that mental simulation might make use of the same trick (Ullman et al., 2017). However, when a physics engine puts an object to sleep, this simply means that the engine assumes that the object remains stationary until acted upon. It does not mean that the physics engine removes the object entirely.

Further, for interacting objects such as those in a falling tower of blocks, it is not possible to ignore objects entirely or put them to sleep. In interacting processes, the accuracy of the simulation depends on the simulation being isomorphic to the real world. If blocks are changed or removed, the predictions made by the simulation will differ dramatically. This leads to the key prediction tested in our first experiment: in interacting multi-object scenes, every object from the initial percept will be represented in each simulation's predicted final state, because every object is necessarily represented and tracked throughout each simulation.

This prediction is unavoidable from the perspective of PMST, but it clashes with well-established characteristics of human cognition. Decades of research has found that people have serious limits on their ability to identify, track, or remember multiple items at the same time. The threshold varies somewhat by domain and with practice, but is often identified as around seven items, plus or minus two (Miller, 1956). Sometimes it is even lower. In general, people can only subitize (accurately count in a glance) a small number of items. Judgments for sets of about one to four items are fast and accurate. But for groups larger than this range, each additional item greatly increases response time and reduces accuracy (Kaufman, Lord, Reese, \& Volkmann, 1949). It seems unlikely that people can accurately and quickly identify each object in a tower of ten blocks (Battaglia et al., 2013), let alone that they can mentally track their simultaneous motions through a complex, rapidly changing, series of physical interactions. The idea of being able to quickly estimate the behavior of sand or fluid by tracking the interactions of model particles, as has been claimed (Bates et al., 2015; Kubricht, Zhu, et al., 2017), seems even less likely. While there are some ways to reconcile the issue - physical reasoning and subitization might not draw on working memory - this inconsistency makes the theory somewhat questionable.

This apparent contradiction between the known limitations of human reasoning and the commitments of PMST is a large part of why we are skeptical of this principle in particular and why we decided to empirically investigate this aspect of the theory.

\subsubsection{Principle 2: Temporal Consistency}

An iterative simulation advances all objects simultaneously. In a simulation of two balls in free fall, each ball accelerates and moves the appropriate amount at every timestep. For the sake of argument, let's assume that the timestep in a simulation is one second long. For each second of the simulation, both balls are advanced the appropriate amount, then the next second is simulated, and so on. Since they always update at the same time, the simulation will have a strong sense of the relative time at which different events occur.

In the absence of extreme uncertainty about the trajectories or objects involved, this step-bystep nature of the simulation means that the order of events is preserved. Preserving the order of events is important for generating accurate simulations and using them to make decisions. When two processes might interact, it is necessary for their simulations to be properly synchronized in order to predict whether and how they interact. 
Suppose, for instance, that you are simulating a person grasping two handles of a box and lifting it straight up. If the simulator advances time on the right hand faster than time on the left hand, then the box will tilt. A synchronous approach keeps errors like this from happening, and ensures that there is no way for one temporal sequence to get ahead of or fall behind another, because they share a common timeline.

The iterative nature ensures that interactions between the objects play out in a predictable way. Simulations need not run in real (i.e., wall clock) time, but they must obey the property that event order remains consistent. In part, this is necessary for a simulation approach to be at all effective. A simulation should never simulate a coffee cup falling off a table before being hit by a person's hand. The hand must hit the coffee cup first to maintain causal and temporal coherence.

Temporal consistency is taken for granted in earlier studies of PMST, and is built into the physics engines that researchers have used. In many cases, maintaining consistency is critical if the simulator is to give results that are correct, or even physically plausible. For example, Ullman, Stuhlmüller, Goodman, and Tenenbaum (2018) used a PMST model to investigate people's ability to infer the properties of multiple pucks interacting on a two-dimensional plane, where the participants saw clips of the pucks interacting and then were asked to estimate properties such as the masses of the pucks and the roughness of certain colored patches of the two-dimensional plane. Based on behavioral data, the authors conclude that features might be learned by running an intuitive physics simulator with different parameter values many times, and accepting the parameters which generate simulations most similar to what was observed. Given how chaotic the collisions of round objects are, especially in combination with the inclusion of additional forces and uncertain parameters, time consistency is critical for a simulation to be useful in making comparisons, just as PMST predicts. If the pucks were not time-locked, then the collisions would not necessarily occur at the correct times. The iterative nature of the simulation is key.

Temporal consistency is also critical for blocktower stimuli, such as those considered in Battaglia et al. (2013). Consider, for example, the situation in Figure 1 and suppose that the simulator advances time for block $\mathrm{C}$ before blocks $\mathrm{A}$ and $\mathrm{B}$. Then in the simulation, first $\mathrm{C}$ will fall off $\mathrm{A}$ and $\mathrm{B}$ leaving them where they are, because their time has not advanced; then B will remain stably on top of $\mathrm{A}$, because $\mathrm{C}$ is gone. ${ }^{3}$ In fact, however, clearly $\mathrm{B}$ will fall to the right simultaneously with $\mathrm{C}$, and probably A will fall over to the left.

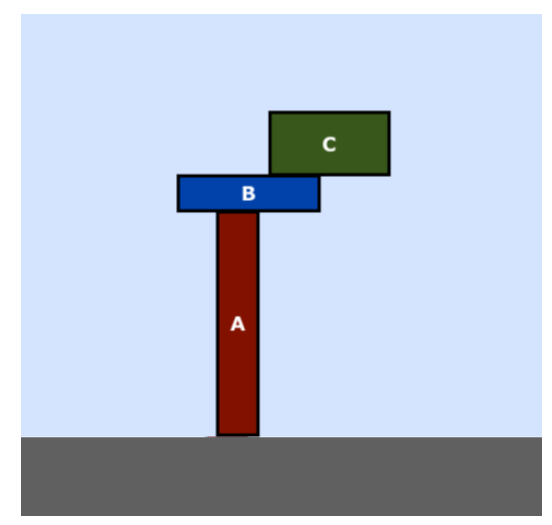

Figure 1: A hypothetical block tower.

In this paper we focus on time consistency between different objects in the same scene, but it is important to note that this is not the only form of time consistency that these models must preserve. For example, Smith, Battaglia, and Vul (2013) present studies of single pendulums. There is only one object in these scenes, so one might suppose that temporal consistency is not an issue. However, this is not the case. While only one object is moving, more than one parameter is being simulated. For a simulator to accurately predict the motion of a bob in free fall, the updates to the velocity of the bob have to be accurately synchronized with the updates to its position, and so even in this case, temporal consistency is a necessary feature of a simulation approach.

\footnotetext{
${ }^{3}$ More precisely, this is a likely outcome of such a simulator. Buggy simulators can be manifest their bugs in different ways, and depending on how interactions are calculated, other outcomes might be possible. But no simulator that violates temporal consistency can give reliable results.
} 
The temporal consistency of events might of course be reversed in some situations due to noise and uncertainty. For example, misestimations of the speed of two marbles might influence which one first hits a barrier. However, such noise is expected to symmetric in most cases such that the errors could go both ways. As long as the noise is unbiased, you should not reliably experience a reversal of your judgment from the reality of the scene.

Of course there are several possible exceptions. In situations where small amounts of noise lead to qualitatively different behavior, predictions from PMST may give systematic time reversals. Consider a game of Cornhole, in which players throw a beanbag at a gently inclined board with a hole in it, with the aim of passing the beanbag through the hole. If the beanbag goes through the hole in the board, it will quickly hit the ground beneath; but if the throw is off by even a little bit, the beanbag will not reach the ground until it has slowly slid down the entire length of the board. Depending on friction, it may not hit the ground at all.

In such cases, noisy initial estimates will lead to different runs of the simulation having greatly different estimates of the order of events in the scene. Some proportion of the outcomes will have the beanbag hitting the ground quickly, in cases where the aim of the player was true. Some other proportion will have the beanbag take a long time to hit the ground, for cases where the board was hit instead. If other events are being tracked simultaneously, then the beanbag's impact with the ground may occur before a given event in some simulations and after it in others. But if the hole were made so wide that it was impossible for a player to miss, all simulations would have the beanbag follow a very similar trajectory, and would report similar relationships for the order of events in the scene.

As a result, systematic reversals can be observed in certain cases where the trajectories of objects are qualitatively uncertain. For objects travelling on predictable paths, however, there should be no such reversals.

You might be wondering why we can say that temporal consistency is a principle of iterative simulation when it degrades under conditions of noise and uncertainty. The important thing to understand is that temporal consistency is a principle of PMST in comparison to any other approach. The use of heuristics, perceptual pattern matching, non-iterative simulation, and other approaches are not expected to show temporal consistency to begin with. Even under conditions of no uncertainty, these approaches admit regular reversals, and should not be expected to reliably preserve the order of events.

For PMST, temporal consistency is a principle (and a feature!) that gets slightly weaker with the addition of noise. With the exception of a small number of perverse cases, it is maintained, and only breaks down under conditions of implausible noise - for example, uncertainty about the position of a ball on the order of magnitude of the ball's diameter. For other approaches, temporal consistency is never a principle to begin with.

\subsubsection{Principle 3: Probabilistic Coherence}

According to PMST, after running multiple (noisy) simulations, the final scene configurations from each simulation are used to make predictions and inferences about the physical world.

Early work, such as Battaglia et al. (2013), proposed that PMST output could be based on a number of aggregation strategies. In the "Will it fall?" task, the output of the model was the average proportion of blocks that fell across the set of the simulation results. For other tasks, the model would compare other numerical or logical predicates (appropriate for the task and judgment) as applied to the set of results.

Another method for making predictions from sets of simulation endstates is Monte Carlo estimation. This has been used to turn the results of simulations into judgments in other PMST models, such as Téglás et al. (2011). In general, probabilistic estimation methods are a common feature of these models (Hamrick et al., 2015), and are especially attractive because of how they match Bayesian models of cognition.

Whether judgments are made via the aggregation of simple predicates, or by more complex Monte Carlo estimation, any systematic way of computing probabilities will conform to the axioms of probability theory. Indeed, this is a key virtue of the approach, and helps to relate the theory to existing Bayesian theories of human inference.

One classic signature of probabilistically coherent reasoning is that the probability of a conjunction of more than one event must always be less than or equal to the probability of one of the components (i.e., $P(A \wedge B) \leq P(A)$ ). But people are not always probabilistically coherent, and in certain cases will estimate conjunctions to be more likely than one of their components (Tversky 
\& Kahneman, 1983). People appear to make these errors in multiple domains. For example, the conjunction fallacy has been observed both in social judgments (the well-known "Linda problem") and in judgments of sequence probabilities generated from rolling dice with red and green sides (the outcome "RGRRR" is judged to be less likely than "GRGRRR", even though the former is contained within the latter).

Conjunction fallacy errors have not previously been observed in reasoning about physical domains, and no reasonable method for estimating the probability of physical events from statistics over of simulation would give rise to such errors. So on the one hand, we see a bias that we might expect to be consistent across domains; on the other, PMST predicts that physical reasoning shouldn't display errors of this type. As before, the cognitive science literature on the subject seems to conflict directly with predictions made by PMST, making an empirical investigation appropriate and informative.

People do, of course, make errors, and there will always be some noise in judgment. Yet even under extreme uncertainty — for example, dealing with a set of totally unfamiliar objects and forces — a simulation will on average maintain the axioms of probability. While the addition of sufficient noise might make a reasoner purely agnostic, this noise cannot introduce bias. One could say that the first moment (i.e. expectation) of the conjunction should always be less than the first moment of either constituent, even if there may be occasional reversals as a result of sampling error. There is no way to make an impossible set of judgments systematically more attractive than a set of judgments that avoid paradox. Occasional confusion will occur due to noise, but this sort of error will not happen regularly, even under conditions of maximum uncertainty.

If likelihood is calculated by tallying the relative outcomes of different random simulations, the conjunction rule will not be systematically violated, because it is impossible to have more random outcomes that include a conjunction than outcomes that include one of the constituent elements.

\section{Experiment 1: Persistence of simulated objects}

Our first study attempts to examine the principle of object persistence in a physical reasoning task. The prediction of PMST is that given an initial scene people should be very accurate at picking out possible future states of the scene and, in particular, should not make mistakes in which they endorse future outcomes that alter the identity, shape, or presence of particular objects in a scene. Alternatively, capacity limits may make it difficult to pick out future world states.

Reasoning about the stability of simple block towers is a prototypical PMST task, both in three dimensions (Battaglia et al., 2013; Hamrick et al., 2016) and in two (Gerstenberg et al., 2017). Given this prior work, in our first study we used a very similar task to maximize the comparability of our results. Much as in previous work, our subjects in this study were asked to make judgments about block towers, each composed of ten brightly-colored blocks which appeared to be sitting on a table. Our materials, however, departed from the previous work in two particular points, both necessary to investigate the claim we were testing.

First, rather than asking participants to make predictions about the collapse of a standing tower, we showed participants one standing tower (the target) and then a set of four collapsed towers and asked them to judge which collapsed option was the result of the target tower falling according to gravity (with one of the four options being the ground truth of running the standing tower through a physics engine). While somewhat unlike previous work with block towers, this kind of judgment task has been used in PMST research on other topics. Kubricht, Zhu, et al. (2017) studied substance dynamics, and in their Experiment 1, participants were asked to indicate which of four sand piles (represented as still images) would result from sand pouring from a funnel at a particular height.

Theoretically speaking, a PMST reasoner should be perfectly able to perform well on post-event judgment tasks. Battaglia et al. (2013) suggest that the theory applies to, "not only objects and their locations, but also ... their likely pasts and futures". In other words, simulations can be used not only to answer questions like, "what will happen?" but also questions like, "what did happen?"

Probing intermediate representational states during mental imagery has a long history. For example, Cooper (1976) found that perceptual processing was facilitated when images where shown to participant at the precise moment they were predicted to have mentally imagined such images. Based on this, people should be very capable of matching an imagined future state to a picture of that state. 
Second, in each trial, the incorrect answers showed a different collection of blocks than in the starting situation. Sometimes blocks were deleted, sometimes new blocks were added, sometimes blocks changed shape or color. In short, the set of objects was not always the same. This is also unlike previous work with block tower materials, but necessary to see if participants were sensitive to such changes.

Because participants were provided with an image of the standing tower in question, previous PMST findings suggest that a reasoner with simulation ability could simply simulate the standing tower forward to generate a set of plausible collapsed tower states. Presumably, the actual result of the tower collapsing would be similar to results generated by the simulation.

A reasoner without access to mental simulation might instead resort to directly comparing the possible endstates to the original tower. We consider in our results several alternative approaches based on structural and perceptual similarity.

\subsection{Participants}

Our stopping rule was designed to collect a fixed number of participants after exclusions. ${ }^{4}$ We ran groups of 9 at a time until the number of participants who meet the criteria reached or exceeded the planned number of participants, which was 100. We recruited 201 participants (71 female, mean age $=33.9, \mathrm{SD}=9.8)$ on Amazon Mechanical Turk (AMT). Following the exclusion criterion outlined below, 101 participants were eligible for our analysis. We analyzed the first 100 (39 female, mean age $=34.0, \mathrm{SD}=10.0$ ). This collection plan and all criteria were preregistered on the OSF.

\subsection{Materials and Procedure}

\subsubsection{Stimuli}

The main stimuli were still images of standing but unstable block towers (the target). Matched with each tower were four still images of possible resting states, i.e. what the tower might look like once it had collapsed.

Each block tower consisted of ten blocks, similar to what has been used in previous research (e.g. Battaglia et al., 2013). The blocks came in three colors (red, blue, and green) and in three shapes (the "cube" in $1 \times 1 \times 1$, the "brick" in $1 \times 1 \times 2$, and the "plank" in $1 \times 0.5 \times 2$; units are relative). Blocks in the initial position of the tower were always aligned with standard coordinate axes.

One of the resting states was always the real result of the target tower collapsing in the physics engine we used to create the stimuli. ${ }^{5}$ The other three options were both incorrect and impossible, in that the collection of blocks in the picture were different in shape, color, or number, from the blocks in the original tower. We call these impossible endstates "foils". For a given critical target tower, the foils were always inconsistent with the original tower in the same way.

The main body of the study consisted of 14 critical questions. These were randomly intermixed with two other types of questions. There were 10 "easy" problems, included as an attention check, which were designed so that the correct answer would be obvious to a participant who was paying attention. For example, in such an item the tower might be entirely composed of green blocks, while three of the answers contained a number of red blocks, rendering them obviously and conspicuously incorrect (e.g., an example easy tower; a conspicuously incorrect answer to the same tower). We also included 4 filler problems for which none of the foils were impossible, being instead the result of small permutations of the starting position.

Question Types There were seven categories of critical questions. For each question, one of the possible answers was correct, and the foils were incorrect and impossible in one of the following ways. In "Change Type" questions, one of the blocks was replaced with a different type of block (i.e. of different dimensions). In "Change Color" questions, one block was switched to a different color. In "Swap Color" questions, two-color towers swapped the colors of all blocks; e.g. all red blocks would become blue and all blue would become red. In "Plus One" questions, an additional block

\footnotetext{
${ }^{4}$ In an earlier preregistration (here), we allowed for a small number of exclusions. However, when we began collecting data for this study we realized that the exclusion rate was much higher than expected. Based on pilot testing, we expected exclusion rates of $15 \%$ or less, but we observed an exclusion rate of $51 \%$. As a result, we stopped data collection and developed a new protocol with a fixed n per experiment after exclusions. See Kennedy, Clifford, Burleigh, Waggoner, and Jewell (2018) for discussion of why the exclusion rate may have been unusually high during the summer of 2018, when the majority of these data were collected.

${ }^{5}$ The PhysX physics engine, through the Unity interface (Unity, 2005).
} 


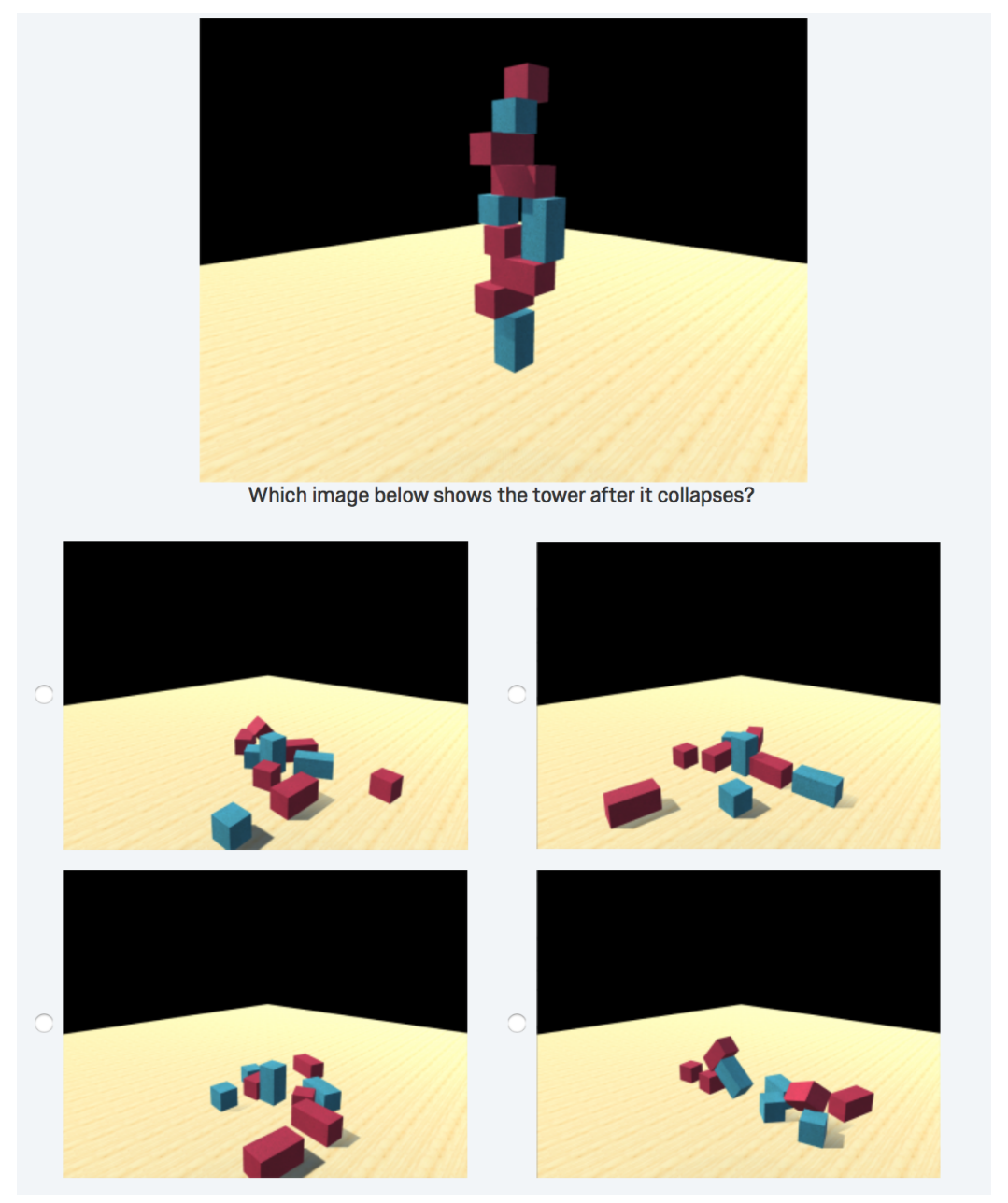

Figure 2: A "Minus One" block tower question, as it appeared to participants. The answer in the upper left is correct; the other three answers are each missing one block.

was included. In "Minus One" questions, one block was missing. In "Minus Two" questions, two blocks were missing. In "Minus Three" questions, three blocks were missing. The study included two questions of each type. The same block or blocks were modified in each of the foils and were always modified in the same way; for example, replaced by the same new type of block or changed to the same new color.

The foils were created by changing the original tower (i.e. deleting a block or changing a block's color), adding some noise (so that the foils were not all identical), and then allowing the simulation to run to rest. Materials were created until there were three foils that were up to the standards of the experiment. These critical standards were (1) blocks never fell outside the viewing area and (2) blocks were never entirely obscured by other blocks.

We also made sure that the foils appeared at least superficially plausible. For example, if a tower consisted of nine green blocks and one red block, we wouldn't create a "Minus One" foil by deleting the red block, as its absence would be salient. This helped ensure that we were examining physical reasoning rather than some sort of more basic logical reasoning (i.e. that a set of nine green elements and one red element doesn't contain zero red elements).

\subsubsection{Instructions and comprehension checks}

Participants read a detailed description of the task. This included several example videos generated from the PhysX materials and example images like those that appeared in the main body of the task. Videos were displayed within the survey software with full controls, so participants were free to pause, reverse, or replay the videos as much as they wanted. Participants were asked to watch each video a few times to familiarize themselves with the blocks and how they would act when 
they fall.

Participants were assured that the simulations were designed to exhibit realistic physical dynamics, that all towers were unstable and would fall over once gravity took hold, and that blocks would never fall outside the viewing area.

Participants then answered eight basic task comprehension questions about the task. Any incorrect answers would restart the instruction sequence. Participants could only move on to the main body of the experiment if they answered all of the comprehension questions correctly on or before their third attempt.

In addition to exclusions, an incentive-compatible design was used to ensure data quality. Participants were told that one question from the main body of the survey would be selected randomly for each participant, and that if they answered that question correctly, they would be given a $\$ 3$ bonus. This was to ensure that participants were equally motivated throughout the study, as any question could be the question upon which their bonus payment would be based.

\subsubsection{Experimental Trials}

In the main body of the study, participants saw images of standing but unstable towers of 10 blocks, the same number as used in previous research (Battaglia et al., 2013; Hamrick et al., 2016). For each tower, they were shown four images of fallen towers (displayed on the same page with the original tower still visible), and asked to choose which one showed the end result of the tower collapsing (Figure 2).

\subsubsection{Follow-up Questions}

Following the completion of the main body of the study, participants were asked to describe the way they completed the relevant tasks using the following prompt: "Roughly speaking, how did you try to solve the problems? Please tell us a little about your approach below." Finally, participants answered demographic questions and were debriefed.

The survey used for this study can be viewed here.

\section{$2.3 \quad$ Results}

\subsubsection{Catch trial analyses}

Of the 201 participants who initially agreed to participate, 42 failed to correctly answer the comprehension questions within 3 attempts. Of those who did answer these questions and moved on to the main body of the survey, 58 answered more than 2 of the 10 easy questions (included to measure attention; see above) incorrectly and were not included in our analysis. This left 101 participants who passed our criteria. We analyzed the first 100, in accordance with our preregistered analysis plan.

\subsubsection{Primary Analyses}

In accordance with our preregistration, we pooled the number of correct answers participants gave on the 14 critical items (Figure 3), and used both a two-tailed one-sample $t$-test and the one-sample "Bayesian Estimation Supersedes the t-Test" (BEST, Kruschke (2013)) to estimate credible intervals for overall performance. The average number of correct answers was 6.33 (SD $=2.37$ ). We calculated a $99 \%$ confidence interval of [5.75, 7.00], and the one-sample BEST gave a $99 \%$ credible interval of $[5.72,6.98]$. Overall performance on these items appears to be better than an "eyes closed" null of $25 \%$ (which would be 3.5 items answered correctly). However, overall performance on the items also appears to be inconsistent with performance as low as $50 \%$ ( 7 items correct), given that the $99 \%$ intervals of both approaches either do not include or border almost exactly upon that value. This is strikingly less than the high levels of performance expected by simulation theories of physical reasoning and the results of past empirical studies Battaglia et al. (2013).

Individual-level analysis Figure 3 shows the distribution of correct answers to the twelve block towers of interest. Notably, 39 of the 100 participants gave the correct answer to fewer than half of the items. Only 3 participants made no errors at all. 
Table 1: Object Tracking Accuracy by Item

\begin{tabular}{|c|c|c|c|c|}
\hline Item Type & Accuracy & $\mathrm{SD}$ & $\begin{array}{l}\text { Corrected }(99.29 \%) \\
\text { Confidence Interval }\end{array}$ & $\begin{array}{c}\text { Corrected }(99.29 \%) \\
\text { Credible Interval }\end{array}$ \\
\hline 1-2. Change Type & .19 & .28 & $.12, .27$ & $.00, .23$ \\
\hline 3-4. Change Color & .40 & .39 & $.29, .50$ & $.28, .50$ \\
\hline 5-6. Swap Color & .30 & .32 & $.21, .89$ & $.20, .38$ \\
\hline 7-8. Plus One & .33 & .32 & $.24, .42$ & $.23, .41$ \\
\hline 9-10. Minus One & .59 & .36 & $.49, .69$ & $.49, .52$ \\
\hline 11-12. Minus Two & .68 & .34 & $.59, .78$ & $.60, .78$ \\
\hline 13-14. Minus Three & .69 & .36 & $.60, .79$ & $.60, .80$ \\
\hline
\end{tabular}

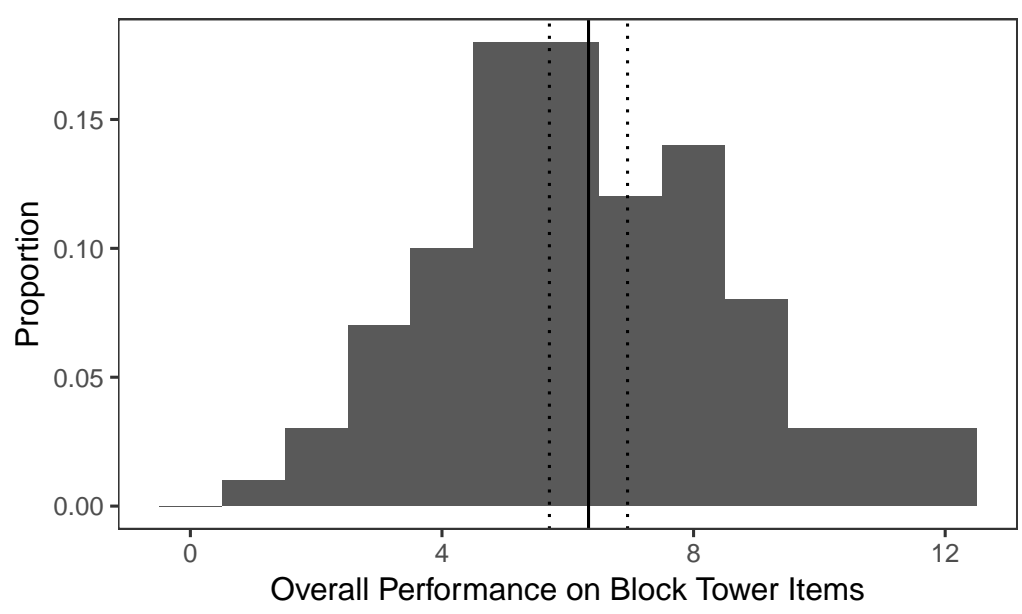

Figure 3: Distribution of Overall N correct (out of 14) in Experiment 1: Multiple Object Tracking. The solid vertical line indicates the mean and the dotted vertical lines indicate the upper and lower bounds of the $95 \%$ confidence interval.

Item-level analysis We also looked at performance by item type. There were seven types of critical items and two items of each type. We combined the two items of each type for each participant and used both a $t$-test and the BEST to compare the empirical mean performance on these items to two null hypotheses; a null of 0.25 , which is the expected performance for the group if all participants did not see the initial configuration of the tower at all, and a null of 0.90 , representing essentially perfect performance with room for some error (motor noise, inattention, etc.). These tests were corrected for multiple comparisons (Bonferroni with $.05 / 7=0.00714$ ) given that there were seven item types. Table 1 contains the means, standard deviations, and $99.29 \%$ intervals (Bonferroni-corrected) from both tests for each of the seven item tests.

First we examined the "eyes closed" null of 0.25 accuracy. Some item types appeared to be consistent with this null. Both intervals for Swap Color and for Plus One include 0.25. The confidence interval for Change Type includes 0.25 , and the credible interval for the same item actually excludes it on the upper bound, indicating performance that is possibly worse than this null. Overall, the results suggest that for these item types, participants perform as poorly as if they were given no information at all.

We also compared item types to a high capability but imperfect null of 0.9 accuracy. No item types appear to be consistent with this null. The highest upper bounds, for Minus Two and Minus Three items, are in the range of .75 (.775-.795). This suggests that even for the items with which the participants had the greatest success, they were still reaching levels of performance well below .90 .

\subsubsection{Modeling}

To explore upon what basis participants may have made their judgments in Experiment 1, we compare several models. The results are presented in Figure 4. Our guiding principle here was that, if participants are able to simulate the falling tower, their judgments should reflect properties 
of the true fallen tower and its similarity to the three foils. Whereas if they are unable to simulate, judgments are necessarily restricted to comparisons between the initial image of the tower and four response options.

For generality, we explored two notions of similarity: (1) Structure similarity. Here we used structural similarity index defined by (Wang, Bovik, Sheikh, \& Simoncelli, 2004). Structure similarity index exploits tries to capture perceived similarity between images by decoupling the luminence, contrast properties from the structural features of the images. (2) Deep Similarity. Here we used a generic deep image similarity API (https://deepai.org/machine-learning-model/ image-similarity). These kinds of computer vision systems, created using deep learning (LeCun, Bengio, \& Hinton, 2015) are currently the best algorithmic methods available for comparing two images.

If people fundamentally do not simulate but rather try to match the final scenes against the initial scene in a more basic perceptual way, we expect that their distribution of responses will reflect some shallower form of comparison between the starting state and the four possible end states. In this case, models Initial State Structural Similarity and Initial State Deep Similarity, which compare the initial state of the standing tower to each of the four response options, would be likely to predict responses.

Conversely, if people are able to simulate forward, at least in some partial or approximate way, their judgments should reflect a comparison of the options against an internal end-of-simulation state representation that reflects the actual fallen tower image but not the foils. In this case, models Post Simulation Structural Similarity and Post Simulation Deep Similarity, which assume participants are matching the response options (imperfectly) against an accurate internal representation of what the fallen tower should look like, would be likely to predict responses.

We also separately considered a simple hand-coded Edit Distance Heuristic, that compares the objects in the scenes with no regard for their positions. This heuristic scores the plausibility of each of the foils as the inverse of an edit distance that simply counts the number of changes required to match the set of objects in the original scene to those in each foil. It therefore makes exactly the same prediction comparing the initial image to the response options as it would comparing the post simulated state to the response options since the simulation does not change the content of the scene but only the positions of the objects. We take a colour change or shape change to constitute an edit distance of 1 and count a missing or additional object as an edit distance of 2 corresponding to its 2 features. Further, we use the smallest edit distance in cases where there is ambiguity about the identity of the objects as in the reverse colours trials (5-6). For this heuristic, the actual fallen tower scene always received a score of zero while the foils all had a negative score according to how many changes in the number and nature of the blocks would be required for them to contain the same objects as the initial scene. For example, for the Reshape 1 trials, all foils had scores of -1 corresponding to the changing of a single block's shape feature while "Minus three blocks" foils had scores of -6 corresponding to the disappearance of both features of all three missing blocks. The catch trial foils had scores that sometimes differed across foils ranging from -1 to -11 , while for the the noise-only trials, all response options had edit scores of zero since they contained the same objects as the initial scene. The full set of edit distances are provided in Table 4 in the Appendix.

Table 2: Experiment 1: Judgment Models

\begin{tabular}{llrrrr}
\hline & Model & $\tau$ & $\log \mathrm{L}$ & Pseudo- $R^{2}$ & $\mathrm{BIC}$ \\
\hline 1 & Baseline & - & -3881.62 & 0.00 & 7763.25 \\
2 & Initial state structural similarity & $>1000$ & -3881.63 & 0.00 & 7766.59 \\
3 & Initial state deep similarity & $>1000$ & -3881.63 & 0.00 & 7766.58 \\
4 & Post simulation structural similarity & 0.04 & -3547.47 & 0.09 & 7098.27 \\
5 & Post simulation deep similarity & 0.55 & -3544.40 & 0.09 & 7092.13 \\
$\mathbf{6}$ & Edit distance heuristic & $\mathbf{2 . 1 7}$ & $\mathbf{- 2 8 3 2 . 0 5}$ & $\mathbf{0 . 2 7}$ & $\mathbf{5 6 6 7 . 4 3}$ \\
\hline
\end{tabular}

For each model, we fit a softmax function with a temperature parameter $\tau$ to maximise the likelihood of producing the count data. Smaller values of $\tau$ correspond to harder maximisation over the model scores. The results are detailed in Table plotted in Table 2 and visualised in Figure 4. Tightness of fit is assessed with McFadden's Pseudo- $R^{2}$ and Bayesian Information Criterion (BIC) is used to compare the relative fits of these models while penalising all but the baseline model for the fitted $\tau$. 
a) Critical trials
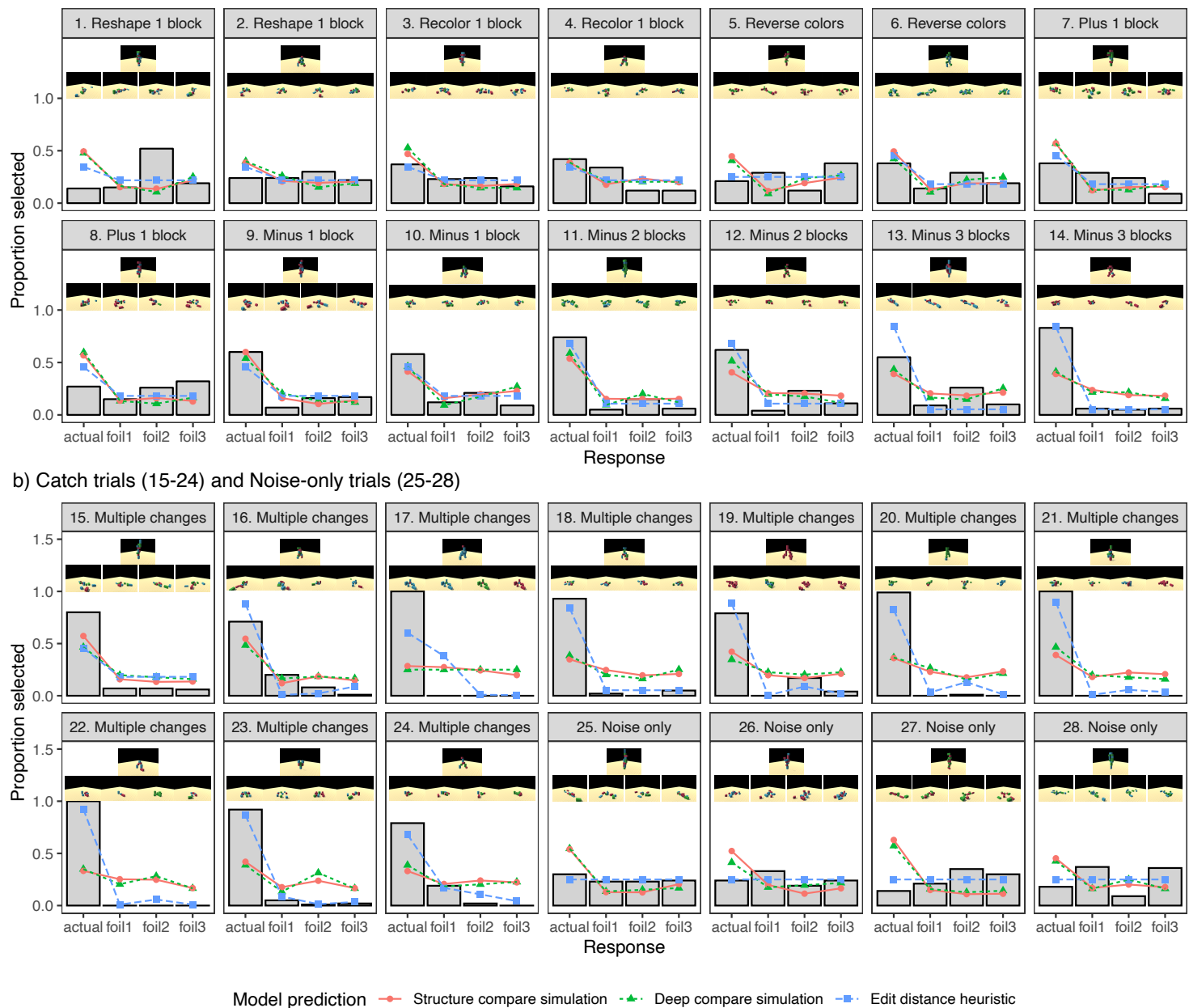

b) Catch trials (15-24) and Noise-only trials (25-28)
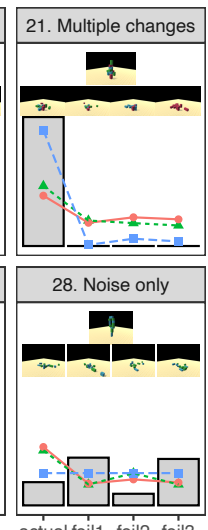

Model prediction $\rightarrow$ Structure compare simulation $\cdots$ Deep compare simulation $\rightarrow$ - Edit distance heuristic

Figure 4: Full results and model predictions from Experiment 1. Grey bars show participant response proportions and colored lines show fitted model predictions for Post simulation structural similarity (red, full), post-simulation deep similarity (green, dotted) and our simple edit distance heuristic (blue, dashed, see Table 2).

From this we can see that neither of the similarity measures provides a close fit to the response counts. Structural and deep similarity to the non-fallen tower have no relationship with the participant response counts, while similarity to the fallen tower had only a small amount of predictive power. The simple Edit Distance Heuristic does considerably better in terms of both Pseudo- $R^{2}$ and BIC, suggesting that participants' responses can in large part be captured by the idea that participants sometimes noticed that objects were missing or had changed in terms of their shape or colour with this detection probability increasing with the number of these changes.

Interrogating Figure 4, we see that on average participants appear to favor a foil over the true scene in some critical trials $(1 ., 5 ., 8$.) and are close to chance for several others $(2-3 ., 6$.). They are also at chance for all noise-only catch trials $(25-28$.). Participants thus fail on a number of trials where where the two simulation-based similarity models succeeded. Interestingly, the structural and deep similarity algorithms are nearly insensitive to trial 17, where only the colors but not positions of the objects changed, while humans and the heuristic found these changes trivially easy to spot.

\subsubsection{Free Response Analyses}

Some previous work has suggested that people may solve physical reasoning problems in multiple ways (Smith, Battaglia, \& Vul, 2018). These proposals have been invoked to explain why people's judgments are full of errors in certain situations and yet highly accurate in others; the explanation offered is that different tasks engage different systems of knowledge. 
If we do have this sort of "diverse toolset", it makes sense that we might apply different tools to different problems. There is nothing, however, stopping a person from becoming confused, or becoming bored, and using an unusual system of reasoning to attempt to solve a particular task. If simulation is one among many approaches, this would suggest that for any particular task, some participants might use a simulation approach, while others use some other strategy to generate an answer.

Because of this issue, we specifically asked participants to use their imagination when solving these problems, in an attempt to encourage them to use a simulation approach. After completing the comprehension questions, and immediately before seeing their first trial, we told participants, "When you see each tower, imagine it falling over, what that would look like, and where the blocks will end up." Despite this, it may have been possible that some participants elected to answer the trials without using simulation.

While we can't know what approach participants actually took, we can ask them approximately how they tried to solve the problems, and see if different endorsed approaches led to different levels of performance.

At the end of the study, we included a free-response question asking participants to describe the way they completed the relevant tasks. Participants saw the following prompt: "Roughly speaking, how did you try to solve the problems? Please tell us a little about your approach below." Three coders who had not been involved in the design of the study or the collection of data were asked to code the free responses into the following categories: 0) No response, Nonsensical response, or "Other" strategy (e.g., "How I thought the blocks will move" ; "none"), 1) Simulation, Visualization, or Imagination (e.g., "I tried to imagine how the blocks would fall when gravity kicked in and made my best guess."), 2) Heuristics or Rules (e.g., "I tried to judge which direction the towers would fall and then look at which blocks are at the top of the tower. I also ruled out impossible color combinations."), 3) Both Simulation \& Heuristics (e.g., "I just tried to visualize how they'd fall and hopefully got close. I'd usually look at the bottom piece first and figure where that'd end up, then the top, then I'd work on the middle.").

Three coders who had not been involved in the design of the study or the collection of data were asked to code the free responses into these categories. In order to conduct subgroup analyses, we first used a best 2 out of 3 approach to resolve disagreements among the coders, and had the three coders manually resolve disagreement for the small number of self-reports where all three coders coded the response differently. The ratings had a Cronbach's alpha of 0.85, indicating acceptable agreement (Kline, 2013).

Of the 100 participants, 19 gave answers that suggested a simulation or visualization approach, 50 said they used specific rules or heuristics, 20 said that they used both simulation and heuristics, and the remaining 11 gave no answer or an uninterpretable answer.

A one-way between subjects ANOVA was conducted to compare the effect of reported approach on overall accuracy on the 14 critical items. There was no significant effect of reported approach, $F(4,95)=0.865, p=0.488$.

\subsection{Discussion}

Past applications of PMST (Battaglia et al., 2013; Hamrick et al., 2016) routinely assume that people track ten simple objects in a tower. Despite this, it appears that on more than $25 \%$ of trials, participants don't notice when two or even three blocks vanish from a tower when judging a possible future state. For more subtle changes, including additions and deletions, performance was even worse.

These behavioral results seems broadly inconsistent with the idea that participants responded by mentally simulating every block before comparing the result of this simulation to the scenes. However, in causally-bound systems, this is a requirement of PMST. While simulators might not represent surface features such as color, tracking the shapes of interacting objects is necessary to predict their behaviour, and tracking every object is necessary to arrive at an accurate guess as to the locations of the objects after falling. Because of this requirement, every object should still be represented in the end states of every mental simulation.

In fact, this is the reason that we included trial types in which we manipulated physically unimportant traits like color. One pattern of results we thought that we might see was a "shoulder", where performance was very good for physically critical traits like shape and mere presence in the scene, while poorer for physically irrelevant traits. Notably, we did not observe this pattern of 
results.

The necessity of object tracking to this model is important because it guarantees that every object from the original scene ends up in every outcome. If people are making judgments about possible endstates by comparing them to the results of simulations, endstates with missing objects should provide a poor match. Strictly speaking, PMST does not appear to make specific claims about how such comparisons would be carried out, but it would be quite strange if multiple deletions did not strongly influence the results of these operations.

In fact, this is exactly what we observe in our modeling. Comparing the behavioral results to the models we explored, it appears that participants' judgments are shaped by a rather noisy ability to check for changes in the objects and their features, with little relation to any hypothetical post-simulation positional state. It is possible that difference between the forward-prediction task used by Battaglia et al. (2013) and this endstate judgment altered the set of cognitive reasoning strategies that participants relied on. However, the current versions of PMST theories don't include any principled set of conditions that predict when simulation is or is not likely be invoked for otherwise identical stimuli.

\section{Experiment 2: Temporal Consistency}

PMST requires that simulations be performed in an iterative fashion. At every time-step, the system applies elementary physical rules to each object in the simulation. This is done recursively; once every object has been updated at time $t$, the system moves on to time $t+1$, updates all objects again, and so on (Battaglia et al., 2013).

This ensures that events will generally occur in the correct order, as long as the approximate trajectory is clear. In this study, we assessed whether people have difficulty predicting the order in which events occur, for physical events with reliable trajectories.

The materials for this study consisted of video clips of events in a simple 3-D world. Participants viewed the first two seconds of several short clips of physical scenes in which two independent physical processes unfolded. For example, the physical processes might be two balls, each rolling down its own series of ramps, or they might be two lines of dominoes falling over. Each physical process followed a predictable trajectory, and we informed participants of this fact.

In each scene, we identified one object in each process (usually "the red ball" and "the blue ball"), and participants were asked to predict which of the two objects would hit the ground first. Participants did not see the outcomes of the video clips, so they had to engage in prospective reasoning in order to make this judgment. The key dependent variable was the proportion of scenes for which participants thought the wrong event would occur first.

\subsection{Participants}

As above, our stopping rule was designed to collect a fixed number of participants after exclusions. We ran groups of 9 at a time until the number of participants who meet the criteria reached or exceeded the planned number of participants, which was 60 .

We collected 78 participants (29 female, mean age $=35.1, \mathrm{SD}=9.8$ ) on Amazon Mechanical Turk. Of these participants, 63 were able to answer basic comprehension questions about the task given three attempts. Of these participants, $0(0 \%)$ answered more than 1 of the 4 easy questions incorrectly and were not included in our analysis. This left us with 63 participants who met our criteria, and we analyzed only the first 60 participants (22 female, mean age $=36.4$, SD $=10.2$ ), as stated in our preregistration ${ }^{6}$.

\subsection{Materials and Procedure}

\subsubsection{Stimuli}

The main stimuli were video clips (example clip) showing the first two seconds of a scene (full version of same scene). Each scene included two key objects, one red and one blue, each involved in its own causal chain, which would eventually lead to each object colliding with the ground.

When colored balls were included, the balls were given a texture with a black "cat's-eye" stripe, so that their rolling motion was clearly visible.

\footnotetext{
${ }^{6}$ The same preregistration as before, on the OSF
} 


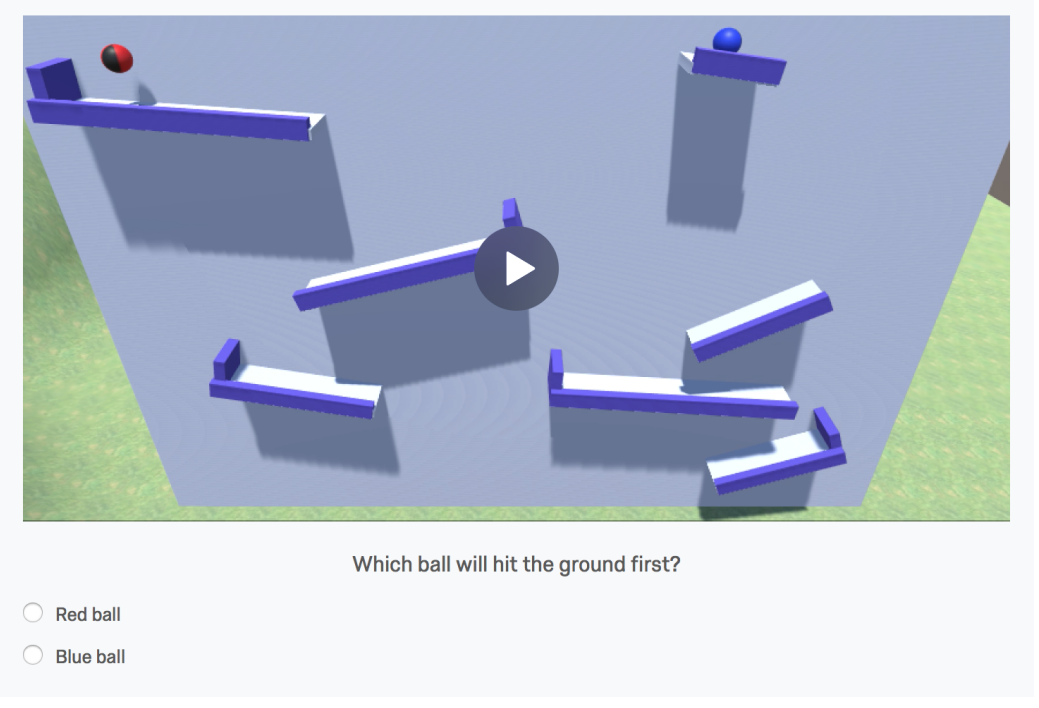

Figure 5: A "marble run"-type question as it appeared to participants. The play button indicates an embedded video.

The critical scenes came in three types:

- "Marble Run" scenes (example), in which two balls roll down several sets of tracks attached to a wall. Each ball has its own set of tracks, and the balls do not interact.

- "Parthenon" scenes (example), in which two balls roll down a series of platforms, steps, and ramps towards the ground.

- "Domino" scenes (example), in which two balls interact with series of dominoes and blocks on top of large cubes, with one of the objects eventually falling off the cubes onto the ground.

Each of these scenes was designed to make the outcome that would occur second seem, at the time of the end of the 2-second clip, more likely to occur first. The object that would actually strike the ground second was moving faster, had gone further, had fewer "obstacles" in its way, etc., or some combination of similar factors. We iterated the design of the scenes based on these heuristics until we believed that pausing at the two-second mark would lead to incorrect judgment of the conclusion. PMST predicts that no such items should exist, as long as the trajectories are clear.

The order of events was unclear to participants, but not actually ambiguous. In the full scenes, the first object always struck the ground at least $2 / 3$ of a second before the second one did, sometimes much earlier. Therefore it was not a matter of degree; the true precedence was never by only a few hundred milliseconds. The times before both objects hit the ground varied somewhat between videos, but all took close to 10 seconds.

\subsubsection{Instructions and comprehension checks}

Participants read a detailed description of the task. This included several example videos of the physics engine we used, and example clips similar to those that appeared in the main body of the survey. Participants were assured that the simulations were designed to exhibit realistic physical dynamics, that both critical objects would always eventually reach the ground, that there were no hidden objects or forces that would interfere, and that everything relevant to the scene was readily visible in the video clips. Videos were displayed within the survey software with full controls, so participants were free to pause, reverse, or replay the videos as much as they wanted. Participants were asked to watch each video a few times so that they would understand how the objects and forces in the scenes work.

Participants then answered 6 simple comprehension questions about the task, and were given three attempts to do so. If they were able to answer these questions correctly, they moved on to the main body of the study. 
In addition to exclusions, an incentive compatible design was used to ensure data quality. Participants were told that one question from the main body of the survey would be selected randomly for each participant. If they answered that question correctly, they were given a $\$ 3$ bonus. This was to ensure that participants were equally motivated throughout the study, as any question could be the question upon which their bonus payment would be based.

\subsubsection{Experimental trials}

In the main body of the study, participants were shown several video clips, showing the first two seconds of a physical scene where two independent chains of events unfold. In each case there were two items of interest, one red and the other blue, and participants were asked to judge which of the two would reach the ground (indicated by a grass texture) first.

The study presented four questions of each type ("Marble Run", "Parthenon", and "Domino"), for a total of twelve critical questions.

There were also four filler scenes, which were designed to be trivially easy.

\subsubsection{Follow-up questions}

Following the completion of the main body of the study, participants were asked to describe the way they completed the relevant tasks using the following prompt: "Roughly speaking, how did you try to solve the problems? Please tell us a little about your approach below."

Finally, participants answered several demographic questions and were debriefed.

The survey used for this study can be viewed here.

\subsection{Results}

\subsubsection{Catch Trial Analyses}

We included four trials which were intended to be very easy, where the order in which the key objects would hit the ground was not misleading (example).

None of our participants (0\%) answered more than 1 of the 4 easy questions incorrectly and were not included in our analysis. This exclusion, combined with the exclusion for failing the instruction comprehension, left us with 63 participants who met our criteria, and we analyzed only the first 60 participants, as stated in our preregistration.

\subsubsection{Primary Analyses}

We used both a two-tailed one-sample $t$-test and one-sample BEST to determine if, on average, participants answered more or fewer than $50 \%$ (6 of 12) of the critical questions incorrectly. Participants answered a mean of 4.77 questions correctly $(\mathrm{SD}=2.55)$, which was less than chance $(6)$, according to both a two-tailed one-sample $t$-test, $t(59)=-3.75,(\mathrm{p})<.001,95 \%$ confidence interval [4.11, 5.42] and a one-sample BEST, 95\% Credible Interval: [4.11, 5.45]. This suggests that participants were not merely guessing on the questions, but actually tended to find the wrong answer more attractive than the true answer. This indicates a lack of time consistency, i.e. significant limitations in subjects' ability to predict the duration of similar physical processes within a single scene.

\subsubsection{Individual-level analyses}

Figure 6 shows the distribution of correct answers to the 12 critical questions (Wickham, 2016). As can be seen, $56.7 \%$ of the participants gave the incorrect answer to more than half of the questions. Every participant made at least two errors - the highest level of performance was ten of twelve correct, achieved by only two participants. Further, $3.3 \%$ of the participants gave the wrong answer on all twelve trials.

\subsubsection{Modeling}

To confirm that there weren't consistent reversals under noise for our stimuli, we ran a series of simulations, using the materials from all twelve scenes. 


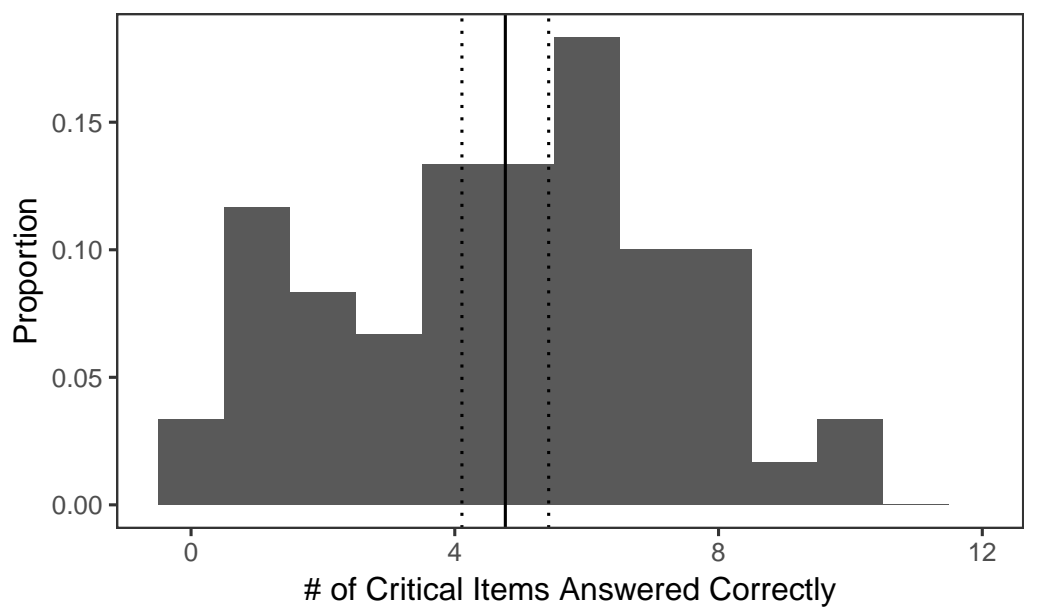

Figure 6: Distribution of overall performance. The solid vertical line indicates the mean and the dotted vertical lines indicate the upper and lower bounds of the $95 \%$ confidence interval.

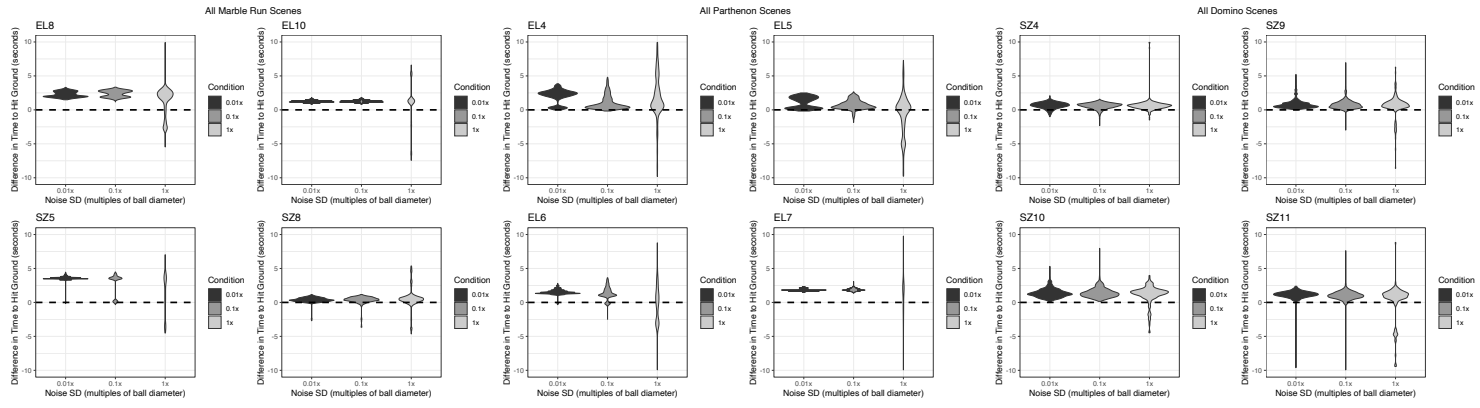

Figure 7: Temporal Consistency Modeling Results. The region above the dashed line indicates simulations where the order of events was the same as the ground truth order of events. We observed temporal consistency for all the Marble Run and Domino scenes. There are minor reversals for two of the Parthenon scenes, but only at the highest level of noise. 
Noise was applied only to the position of the objects that started the chain of events in the scene. The different scene types had different critical objects, but all three used a red and a blue ball to set of their chains of events, so adding noise to these objects alone allowed us to keep the introduction of noise consistent across the three scene types. We used three levels of noise. The noise was normally distributed with a standard deviation of $0.01 \mathrm{x}, 0.1 \mathrm{x}$, and $1 \mathrm{x}$ the diameters of the starting objects. ${ }^{7}$ It's important to note that $1 \mathrm{x}$ diameter is an implausibly large amount of noise that often caused balls to spawn well outside of the scene or even underneath the ground — we included this extreme noise condition for comparison. Marble Run scenes involve objects interacting essentially in what is essentially a fixed plane, and so for these scenes, we added noise only in the $\mathrm{X}$ and $\mathrm{Y}$ dimensions. In the other two types of scenes, noise was added in all three dimensions.

Each combination of scene and level of noise was run through 1,000 simulation trials. In each trial, the time (in seconds) at which each critical object hit the ground was recorded.

Trials where one or both of the target objects never reached the ground were excluded from the analysis, for two reasons. First, we assured participants that both critical objects would always reach the ground eventually, so they should have excluded such hypotheticals from consideration. Second, it's hard to talk about which object hits the ground first when one doesn't hit the ground; so much more so if nether of them hits the ground at all! These cases included trials where both objects appeared outside of the scene or under the level of the ground due to extreme levels of starting noise.

Figure 7 shows the result of all simulations. The Marble Run scenes show no reversals up to the highest levels of noise, suggesting good temporal consistency. The Parthenon scenes show two very minor reversals (EL5 and EL6) at the absolute highest amount of noise. However, the reversals are very slight, as we can see by the extremely wide distributions of the impact times. Further, noise with a standard deviation equal to the diameter of the objects is almost certainly greater than perceptual uncertainty as to position. The Domino scenes show no reversals up to the highest levels of noise, suggesting good temporal consistency.

Overall, there are no reversals at reasonable levels of noise, and only very small, unreliable reversals at the highest level of noise. Notably, these minor reversals are limited to only one of our three scene types, leaving the other two types with entirely reliable temporal consistency.

These simulations are incompatible with the behavioral results. As a result, we reject the hypothesis that people are using mental simulation with noise to answer this task.

\subsubsection{Free Response Analyses}

The same free-response question as appeared in the previous study ("Roughly speaking, how did you try to solve the problems? Please tell us a little about your approach below.") was asked at the end of this study. The same three coders coded these responses according to the system described above. The ratings had a Cronbach's alpha of 0.74, indicating acceptable agreement (Kline, 2013). For the purposes of subgroup analysis, disagreement between the raters was resolved in the same way as described above.

Examples of responses by type:

0. No response, Nonsensical response, or "Other" strategy

- "solve the problem of the all person speaking in the solve the problems"

1. Simulation, Visualization, or Imagination

- "I tried to imagine what would happen going forward and then went with that vision to make an estimate."

\section{Heuristics or Rules}

- "The ones that were closest to the ground. In the block ones, it would probably be how many blocks feel [sic] down."

3. Both Simulation \& Heuristics

\footnotetext{
${ }^{7}$ Focal objects were different scales in different scenes, which is why we describe the noise in terms of object diameter instead of absolute units.
} 
- "Just tried to imagine how the scene would proceed, looking at angles and drops and comparing speeds when the videos stopped."

Of the 60 participants, 8 gave answers that suggested a simulation or visualization approach, 35 said they used specific rules or heuristics, 8 said that they used both simulation and heuristics, and the remaining 9 gave no answer or an uninterpretable answer.

To determine if there was an overall impact of approach, a one-way between subjects ANOVA was conducted to compare the effect of reported approach on overall accuracy on the 12 critical items. We found no effect of reported approach, $F(3,56)=1.450, p=0.238$.

The majority of participants claimed to use a heuristic approach to solve these problems. Given the small number of participants reporting other approaches, we didn't perform any further subgroup analyses.

\subsection{Discussion}

The scenarios used in this experiment have been designed so that random noise in the parameters will not qualitatively change behavior, as demonstrated in our modeling. Therefore, PMST should preserve temporal consistency, and so it predicts that there should be no cases where the order of events is reversed in a prediction, so long as overall trajectories are predictable.

In this study, participants saw two processes with guaranteed trajectories, and were asked to estimate which process would complete first. Overall, participants reversed the order of the events in their predictions, predicting that the event that truly occurred second would occur first, and did so more often than chance.

Admittedly, the scenarios used in this study were deliberately designed to be misleading. If we were to imagine the (hypothetical) space of all possible scenes, it is likely that very few of these cases would prompt the reversals in judgment we observed. But while it is true that our scenes are in some sense non-representative, this does not affect the claims we are making. The design of PMST suggests that no items showing such reversals should exist, barring major uncertainties in trajectory, etc. That there exist any items where this kind of reversal is consistently found is strong evidence that PMST is not the approach being used to make these judgments. More broadly, these results are incompatible with any iterative simulation theory.

Instead, we should be looking for a theory which does allow for reversals of this sort, in certain difficult cases like the ones in our materials. We note that in these cases, people can easily predict what is going to happen on each side of the scene, even though both involve a long causal chain of events. However, participants perform poorly at estimating how long the processes will take, even comparatively. This is a feature that is very characteristic of qualitative reasoning systems (Bobrow, 1984) but not at all characteristic of simulation in a physics engine.

Given that many of our participants' free response answers included appeals to heuristics such as the relative speed of the objects, the number of obstacles in their way, and their distance from the ground, a simple additive model combining this information might provide a good fit for judgments on this sort of task. This kind of investigation provides a promising avenue for future research.

\section{Experiment 3: Probabilistic Coherence}

When making predictions about a physical scene, a key claim of PMST is that judgments reflect probabilistic inference, estimated via repeated stochastic runs of the simulation (Battaglia et al., 2013). As such, people's physical judgments should approximately obey the laws of probability theory. Conjunction fallacy errors, where people rate a joint probability (A \& B both occur) as more likely than the marginal probability of one of the components (e.g. A occurring at all) is logically contradictory because there is no way for the joint probability to be larger than either of its components. At most, it will be equal to the smaller component.

In the cognitive domain, this is often known as the "Linda Problem", because of an early example in which participants judged a hypothetical individual named Linda as more likely to be both a bank teller and a feminist than to be a bank teller in general (Tversky \& Kahneman, 1983). To test this commitment of PMST, in this study we assessed if people fall prey to judgment errors in the style of the conjunction fallacy for physical reasoning problems.

To test this, we created simple 2-D worlds, in which a small number of objects interacted with one another in the presence of gravity. We chose this design to remain similar to the materials used in some previous PMST work (Smith et al., 2017; Ullman et al., 2018). 
For eight critical trials, participants saw the first $2 / 3$ second of the events unfolding. Then they were asked a simple question about what would happen to some of the objects after the events played out more fully. Unbeknownst to participants, they were shown each of the eight critical trials two times. For each video clip, on one showing they would be asked to rate the likelihood of a single outcome $(\mathrm{X})$, and the other showing they would be asked to rate the likelihood of the same outcome and its conjunction with another outcome (X \& Y). We planned to examine whether participants rated the conjunctions as more likely than their components.

These results, as well as some additional studies extending the main findings, have previously been published as Ludwin-Peery, Bramley, Davis, and Gureckis (2020). They are extended here with new modeling work.

In particular, we are interested in using a modeling approach to rule out a specific alternative explanation for these results. A perennial concern in research on the conjunction fallacy is that participants might be interpreting the questions not as literally written, but in line with conversational norms (Gigerenzer, 1991, 1996). People expect statements in conversation to be informative, truthful, relevant, and clear (Grice, 1991), but conjunction questions sometimes violate these expectations, leading participants to answer a slightly different question than the one intended.

Of specific concern here is the conditional. It's possible that participants might interpret "How likely is it that the cannonball will hit the pink sphere, and then the pink ball will end up on the grass?" not as p(grass \& sphere) but rather as p(grass|sphere), something like "Given that the cannonball hits the pink sphere, how likely is it that the pink ball will end up on the grass?" Our other work on this subject (Ludwin-Peery et al., 2020) has provided empirical evidence against this alternative explanation, but here we plan to use modeling to more directly evaluate whether of not this is a viable interpretation; essentially, are participants' answers consistent with estimating $\mathrm{p}($ grass $\mid$ sphere $)$ ?

\subsection{Participants}

As above, our stopping rule was designed to collect a fixed number of participants after exclusions. We ran groups of 9 at a time until the number of participants who meet the criteria reached or exceeded the planned number of participants, which was 60 .

We collected data from 90 participants (28 female, mean age $=33.6, \mathrm{SD}=9.8$ ) on Amazon Mechanical Turk (AMT). Following the exclusion criteria outlined below, 62 participants were eligible for our analysis. We analyzed only the first 60 participants (18 female, mean age $=34.2$, $\mathrm{SD}=9.7$ ) in line with our preregistration ${ }^{8}$.

\subsection{Materials and Procedure}

\subsubsection{Stimuli}

The main stimuli were video clips, $2 / 3$ of a second long, in which two round objects (a pink "sphere" and a gray "cannonball") interacted in a 2-dimensional world. This world included gravity and some stationary objects. There was always "ground" on the bottom edge of the scene, with a green section representing grass on top, and usually one or more boxes resting on the grass. There was always a hole in the ground, wide enough for either object to potentially fall into.

Over the course of each clip, the gray cannonball would travel in a parabola across the screen, while the pink sphere would fall under the influence of gravity (Figure 8).

We had certain criteria in mind when creating these videos. In each scene, the pink sphere fell above the hole, so that it appeared that it might fall in without any interference. Even so, we placed it close enough to the edge of the hole that there would be some chance it would bounce off the edge and not end up in the hole. (Indeed, one of the training videos seen by all participants showed the sphere "improbably" bouncing off the rim and escaping the hole.) The cannonball's position and trajectory were chosen so that the cannonball seemed likely but not certain to collide with the sphere on its way towards the hole. The position of other objects, like boxes, were chosen to suggest that, if the cannonball did hit the sphere, that the sphere would end up on the grass. We tried to make it seem unlikely that, in the event of a collision, the sphere would land in a box or bounce off a box and end up falling in the hole regardless.

\footnotetext{
${ }^{8}$ The same preregistration as before, on the OSF
} 


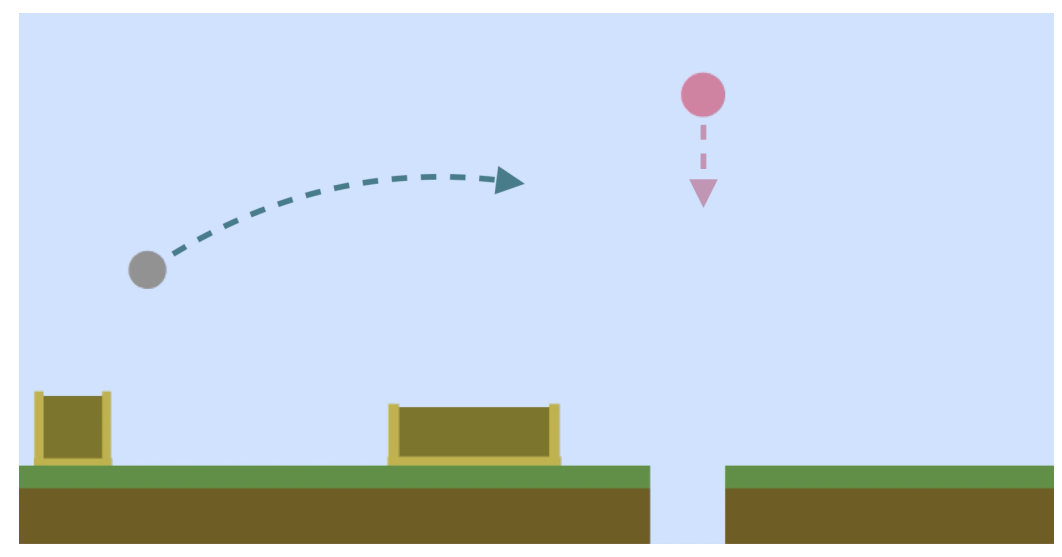

Figure 8: An example scene. Dotted arrows indicate approximate motion over the $2 / 3$ second clip.

\subsubsection{Instructions and comprehension checks}

Participants read a detailed description of the task. This included several example simulation videos from the physics engine we used (Unity, 2005) and example clips like those that appeared in the main body of the task. The example videos included many forms of inter-object interactions, including collisions, and participants were allowed to watch these videos as many times as they wanted. Videos were displayed within the survey software with full controls, so participants were also free to pause the videos at any point. Participants were informed of the nature of the clips and we explained how to report the rating of the likelihood of possible events.

Participants then answered seven comprehension questions about the task. Incorrect answers to any of the questions would restart the instruction sequence. Participants could only move on to the main body of the experiment if they answered all of the comprehension questions correctly on or before their third attempt (failure to meet this standard excluded 16 of the 90 participants who initially agreed to participate).

\subsubsection{Experimental Trials}

In the main body of the study, participants saw several simple physical scenes in which a pink "sphere" fell above a hole in a grassy field, and a gray "cannonball" traveled across the scene in such a way that it could potentially collide with the pink sphere (Figure 9; example video here). One object was called a cannonball and the other was called a sphere, so that it would be less likely that participants would confuse them based on the written description. The scenes differed from one another in several minor ways, including the exact speed and position of the objects, size of the hole, and the presence or absence of one or more boxes on the grass. Each video ended after approximately $700 \mathrm{~ms}$, well before the cannonball could possibly intersect with the pink sphere's path, leaving ambiguity about the outcome of the scene. For each scene, participants were asked to estimate the likelihood of a particular prompted outcome (e.g., "how likely is it that the pink sphere will end up on the grass?"), and express that estimate as a percentage ranging from $0 \%$ to $100 \%$ in $1 \%$ increments.

Eight of the scenes were considered "critical", and the answers to these provided our primary dependent measure. Unknown to participants, each critical scene appeared twice, for a total of 16 critical trials. Half of the scenes that appeared twice were mirrored horizontally in their second appearance.

For each scene that appeared twice, in one appearance participants were asked the question, "How likely is it that the pink sphere will end up on the grass?" and in the other, "How likely is it that the cannonball will hit the pink sphere, and then the pink ball will end up on the grass?" Scenes did not repeat until after several filler scenes were presented and completed.

For the filler scenes, participants were asked questions unrelated to the outcomes of interest used in the critical trials, such as the likelihood that the cannonball might end up in one of the boxes or in the hole. Pilot research had indicated that separation by a few filler scenes was sufficient to prevent participants from explicitly recognizing the repetition. Two of the filler trials were considered "catch trials" because they asked about a trivially obvious outcome (e.g. the cannonball 


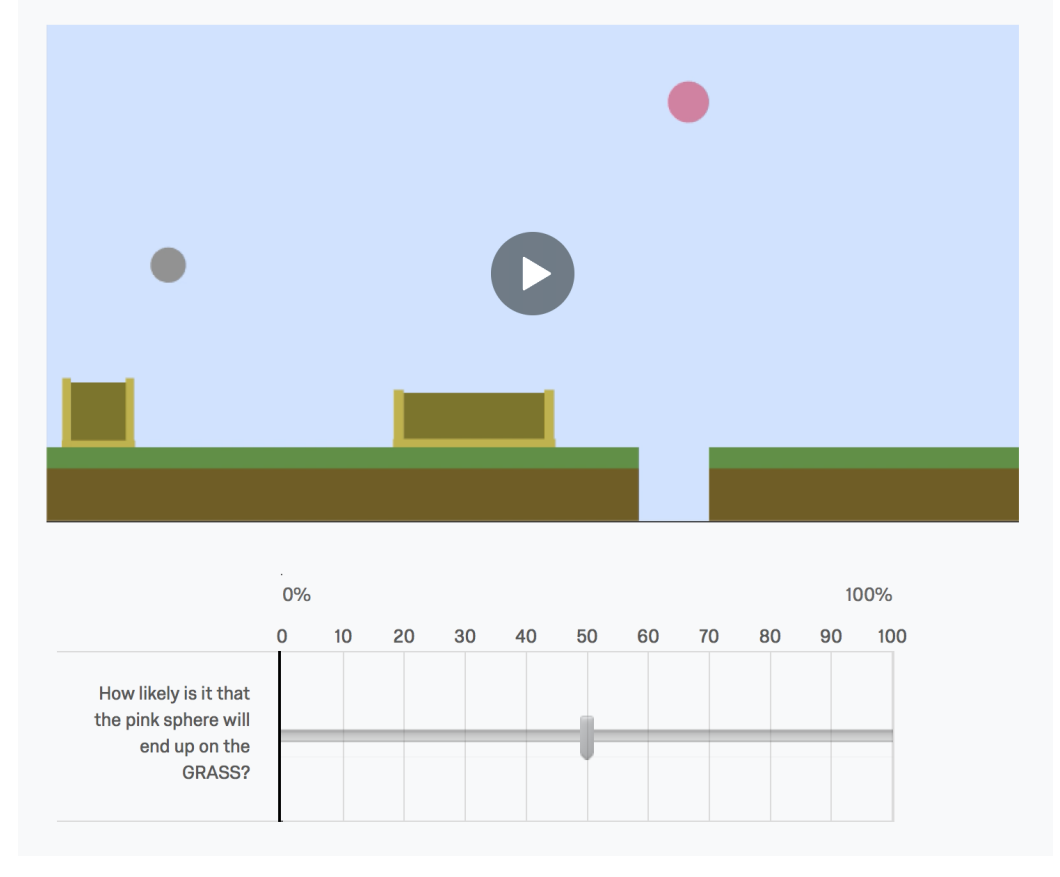

Figure 9: A conjunctive reasoning question as it appeared to participants. The play button indicates an embedded video, which is two-thirds of a second long.

had already missed the sphere and couldn't possibly collide with it). These were used as attentional checks as discussed below.

\subsubsection{Follow-up Questions}

Following the completion of the main body of the study, participants were asked in an open-ended way to describe how they believed they completed the tasks using the following prompt: "Roughly speaking, how did you try to solve the problems? Please tell us a little about your approach below." Similarly, we asked several open-ended questions intended to determine whether or not participants had noticed that some of the scenes appeared twice with different questions. Finally, participants answered several demographic questions, gave free-response feedback, and were debriefed.

The survey used for this study can be viewed here.

\subsection{Results}

\subsubsection{Catch trial analyses}

Two filler questions had trivially obvious outcomes (e.g. the cannonball had already missed the sphere and couldn't possibly collide with it; video here), the outcome of one seeming nearly certain and the outcome of the other seeming nearly impossible. When answering these questions, $12(16 \%)$ reported that the near certain outcome was less than $90 \%$ likely or that the near impossible outcome was more than $10 \%$ likely, and were not included in our analysis. This exclusion, combined with the exclusion for failing the instruction comprehension, left 62 participants for our main analysis (we analyzed the first 60 due to our preregistered sample size).

\subsubsection{Primary analyses}

We averaged the rating difference scores (conjunction probability - sole probability) for each participant for each of the eight critical scenes. Positive values on these difference scores indicate that participants rated a conjunction as more likely than the constituent sole probability, which is a form of the conjunction fallacy. The average rating difference score was $7.29(\mathrm{SD}=13.07)$, which was reliably greater than zero, according to both a two-tailed one-sample $t$-test, $t(59)=4.32, p$ $<.001,95 \%$ confidence interval [3.92, 10.67], and a one-sample BEST (Bayesian Estimation Su- 

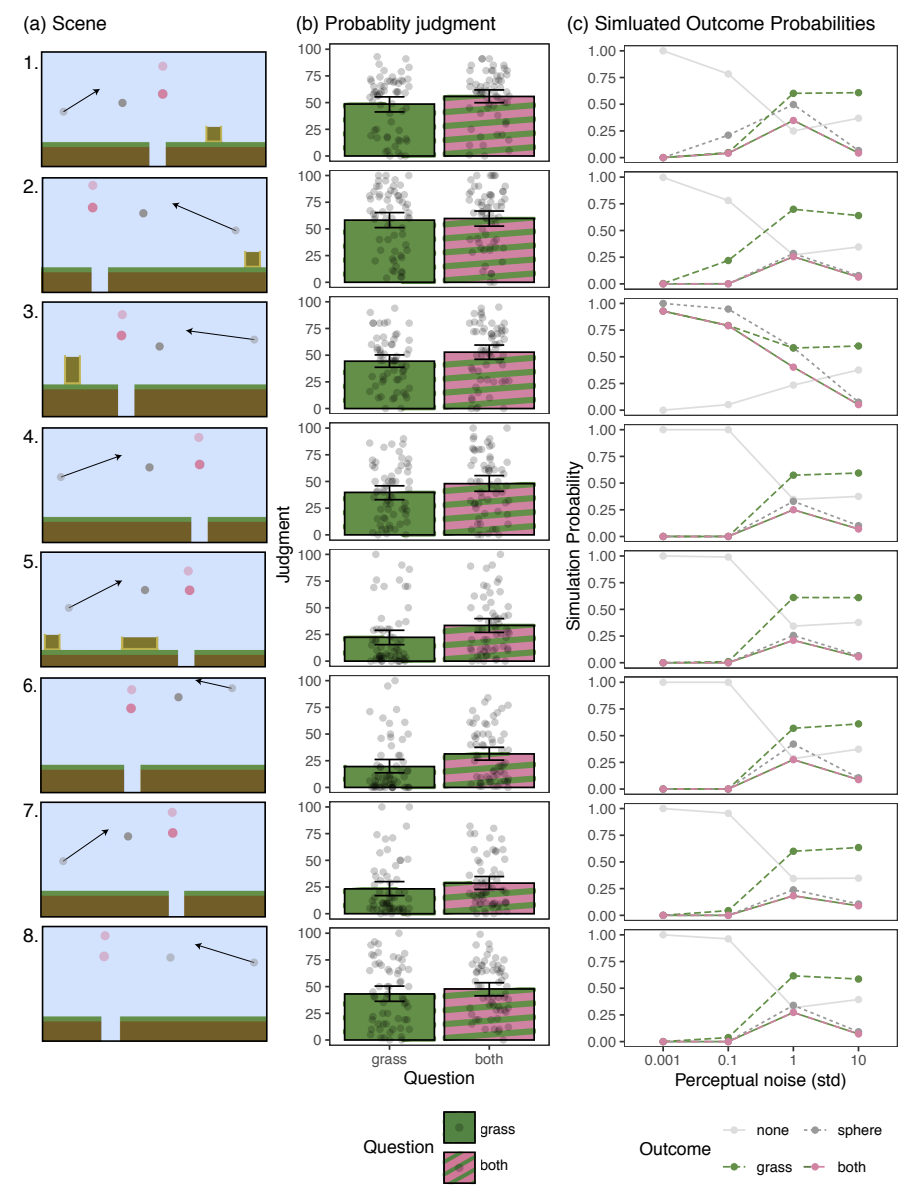

Figure 10: a) The state of each test scene. Faded objects depict the objects at the start of the clip, including the velocity vector for the cannonball (black arrow), and solid objects indicate positions at the moment where the clip is paused. The pink sphere always dropped vertically from a stationary start. b) Participants' judgments of the probability the cannonball will end on the grass (green) and the chance it will collide with the pink sphere and end up on the grass (green and pink stripes). c) The probabilities of the four possible outcomes according to probabilistic simulation analysis having applied four different levels of noise to starting positions ranging from $0.001 m^{2}$ to $10 m^{2}$.

persedes the t-Test, BEST, Kruschke (2013)), 95\% Credible Interval: [4.06, 10.79]. This suggests that, on average, participants were inclined to commit the conjunction fallacy in a physics domain.

\subsubsection{Modeling}

To further investigate these results, we compared participants' judgments to the simulated probabilities, depicted in Figure 10 (assuming SD = 1 for noise). Averaged participant judgments for both $\mathrm{P}$ (grass) ("How likely is it that the pink sphere will end up on the grass?") and P(grass \& sphere) ("How likely is it that the cannonball will hit the pink sphere, and then the pink ball will end up on the grass?") are highly correlated across Trials $(r=.97)$ and moderately positively correlated with the requisite simulated probability, $r=.59$ for the single $\mathrm{p}$ (grass) probability and $r=.62$ for the joint $\mathrm{P}$ (grass \& sphere) probability.

Critically, judgments of the likelihood of the conjunction (grass \& sphere) are more correlated with the simulated joint probability $\mathrm{P}$ (grass \& sphere) $(r=.62)$ than with the simulated conditional probability $\mathrm{P}$ (grass $\mid$ sphere) $(r=.24)$.

We fit mixed effects models predicting participant judgments for grass\&sphere from the simulated probabilities with random participant means using the lmer function from the lme4 package in R. We found a significantly better fit for a model with the simulated joint probability $\mathrm{P}$ (grass \& sphere $)(\mathrm{AIC}=4535$, Fixed effect $( \pm \mathrm{SE})=103.5 \pm 18.1)$ than a model using the simulated conditional probabilities $\mathrm{P}($ grass $\mid$ sphere $)\left(\mathrm{AIC}=4562\right.$, Fixed Effect $\left.=35.6 \pm 16.8, \chi^{2}=27.211, p<.001\right)$. 
However, both the simulated joint $\mathrm{P}$ (grass \& sphere) and conditional $\mathrm{P}$ (grass | sphere) probabilities contribute independently to predicting grass\&sphere judgments $(\mathrm{AIC}=4497$, Fixed Effects joint $172.6 \pm 20.3, t=8.5$, conditional $\left.=117.5 \pm 18.3, t=6.4, \chi^{2}=39, p<.001\right)$. A model predicting grass\&sphere judgments with fixed effects for joint $\mathrm{P}$ (grass \& sphere), conditional $\mathrm{P}$ (grass $\mid$ sphere) and participants' own singular grass judgments does better still $(\mathrm{AIC}=4462$, Fixed Effects joint $=122.4 \pm 21.3, t=5.7$, conditional $=17.8 \pm 18.9, t=4.1$, grass judgment $=0.27 \pm 0.043, t=6.2)$.

\subsection{Discussion}

In this study, participants making judgments about outcomes in physical processes routinely predicted that conjunctions were more likely than one of their constituent events. Generally, these results were not predicted by PMST, and this type of error contradicts a central part of the theory. PMST states that judgments about the outcomes of physical processes are made by aggregating over the result of multiple noisy runs of a simulation (Battaglia et al., 2013). Because there is no way to end up with more outcomes containing a conjunction event than containing a particular constituent event of that conjunction, these results provide strong evidence against this model.

The discovery of conjunction fallacy errors in the physical domain also has interesting general implications. First, they provide a strong argument against explicit claims that the conjunction fallacy does not apply in the physical domain. For example, the philosopher Michael Strevens argues in his book Tychomancy (2013) that while people make errors in some domains, they will not do so for physical events. According to Strevens, despite come consistent errors in some forms of inductive reasoning, people have a "domain-general genius at inferring physical probabilities." Our version of the conjunction fallacy, in which we collected judgments of physical probabilities, demonstrates that there are in fact important limitations on this genius.

The results of this study also appear to support the original interpretation of the fallacy made by Tversky and Kahneman (1983). There have been some attempts to argue that the original conjunction fallacy results were the result of issues stemming from pragmatics or polysemy in the phrasing of the questions. Hertwig and Gigerenzer (1999) ran a series of studies to investigate this possibility. They found results that they suggest indicate that participants appear to commit the conjunction fallacy because of the way they interpret the term "probability". Instead of taking the term in its mathematical sense, they suggested that participants instead interpreted it as requesting a typicality or possibility judgment.

The results of the experiments reported here, however, are resistant to this alternative explanation. In our studies, we explicitly asked for mathematical judgments, expressed in percent likelihood. Further, while people may be a more or less typical example of a social category (as argued by Hertwig and Gigerenzer), there is no sense in which a scene could be a "better example" of an outcome except insofar as the outcome is more or less likely.

\section{General Discussion}

Simulation-based explanations of physical reasoning ability, such as PMST, offer a powerful and flexible model, which provides a good fit to behavioral data in many cases. In this paper, we chose to investigate this theory further, asking the following questions: Are there limits on the use of simulation as a strategy? And how do the predictions made by this model stand up to empirical investigation?

Across three studies, we found serious contradictions between the predictions made by PMST and human behavior, across three distinctly different tasks.

First, when trying to identify the resting state for an unstable tower of 10 blocks, participants have great difficulty distinguishing between the true set of blocks and sets that differed because of changes of color, changes of dimensions, additions, or deletions. Even when three blocks were missing from the incorrect options, one of these distractors was chosen more than $25 \%$ of the time. PMST suggests that this should not happen; every object must be simulated through the course of a scene, so every object should be present and available for comparison in the result of those simulations. Furthermore, modeling suggests that participant behavior in this experiment is consistent with the application of a simple heuristic, and inconsistent with the comparisons of the observed endstates to simulated endstates.

Second, when judging the order of events in a scene with highly predictable trajectories, but with potentially misleading cues, participants consistently made incorrect predictions about the 
order of events. This is an outcome which shouldn't happen when judgments are made using an iterative simulation, because for each unit of time, each object is advanced exactly once, preventing reversals.

Third, participants consistently committed the conjunction fallacy (Tversky \& Kahneman, 1983) when reasoning about simple physical scenes, a result that contradicts the claims of PMST about how prospective judgments are made about physical scenarios. Modeling confirmed that this should not be observed when simulations are aggregated across multiple runs, and ruled out a pragmatics-based alternative explanation.

In all cases, the observed evidence was strongly inconsistent with PMST, and some of these findings are inconsistent with simulation explanations of physical reasoning in general.

\subsection{Criticism and Controversy}

\subsubsection{Are different mechanisms used for different tasks?}

Some researchers have proposed that different cognitive mechanisms are used for different sorts of tasks. For example, Smith et al. (2018) compared subjects' behavior on a number of similar tasks and concluded that "the contrast between rich and calibrated versus poor and inaccurate patterns of physical reasoning exists as a result of using different systems of knowledge across tasks." The implicit point of such arguments is that the difference between experiments that find support for simulation accounts of physical reasoning, and those that don't, has to do with the task.

Findings such as these are certainly interesting, in that they establish that sometimes the reasoning mechanism changes based on the task. But, over the literature as a whole, there does not seem to be any systematic relation between the kind of task and the ability of participants to find the correct answer. At most we can see the difference between correct and incorrect answers; or more-correct and less-correct answers.

In the vast majority of experiments in both directions, the actual task is to give some kind of verbal answer to a verbal question. Consider Table 3, which collects and reviews a number of physical reasoning results. The table describes the task participants are asked to do in each case, and estimates the degrees of freedom / number of parameters for the system in question.

A strong version of the claim that different mechanisms are used for different tasks is that when acting in the physical world (or simulated physical world) people can call on powerful simulation mechanisms to provide quick and accurate judgments and behaviors, whereas if they are just answering questions posed and answered verbally, they don't recruit these abilities, and give much less accurate answers, based on some other system. If this were the case, we would expect to see highly accurate, simulation-consistent behavior on tasks that have a behavioral element or that engage the simulation system (perhaps by posing the task using visual materials, rather than a strict verbal description). We would also expect to see, that when participants give verbal estimates in response to purely textual or otherwise very information-poor questions, they give less accurate, simulation-inconsistent answers. This is the main thrust of the research by Smith et al. (2018); for example, in Experiment 1 they compare accuracy on a nonbehavioral measure (drawing the path of a cut pendulum) to accuracy on two behavioral measures (cutting the string or catching the ball), within a small 2-D game world.

However, the experiments cited in Table 3 do not conform to that pattern. Many cases in which the task was verbal are consistent with simulation (e.g. Hegarty (1992)), and many cases of behavioral tasks give results which are inconsistent with simulation (e.g. Proffitt et al. (1990)). The question becomes why one would suppose that predicting whether a tower will fall (accurate answers in Battaglia et al. (2013)) is a "different domain of behavior" from predicting which of two wheels will roll faster (inaccurate answers in Proffitt et al. (1990)) or our results from Experiment 1 .

As this literature review shows, the field often serendipitously discovers errors without being able to predict them in advance. However, in this paper we combined predictions of PMST with the broader literature on human cognitive and perceptual limitations and were able to design stimuli that we expected might violate the predictions of PMST.

\footnotetext{
${ }^{8}$ The simulator used between 1 and 100 particles. For each particle, the simulator tracked position (3 parameters), velocity (3 parameters), and pressure (1 parameter). Density and mass are also parameters of particles, but these are presumably constant over time for an incompressible fluid.

${ }^{10} 40,000$ particles, with position and velocity tracked for each particle.
} 


\begin{tabular}{|c|c|c|c|c|c|}
\hline Citation & Exp. & Physical Situation & Task & $\begin{array}{l}\text { Explained by } \\
\text { Simulation? }\end{array}$ & $\mathrm{DoF} / \mathrm{NoP}$ \\
\hline Bates et al. (2015) & & $\begin{array}{l}\text { Liquid flowing } \\
\text { around fixed obstacles } \\
\text { into two basins }\end{array}$ & $\begin{array}{l}\text { Show with a slider } \\
\text { the fraction of liquid } \\
\text { in each basin }\end{array}$ & Yes & $\sim 10^{24} / 8-800^{9}$ \\
\hline \multirow[t]{3}{*}{ Battaglia et al. (2013) } & 1,3 & Tower of blocks & $\begin{array}{l}\text { Verbally predict } \\
\text { whether it will fall }\end{array}$ & Yes & $\sim 120$ \\
\hline & 2,4 & Tower of blocks & $\begin{array}{l}\text { Indicate } \\
\text { direction of fall }\end{array}$ & Yes & $\sim 120$ \\
\hline & 5 & $\begin{array}{l}\text { Bump into table } \\
\text { supporting towers } \\
\text { of blocks }\end{array}$ & $\begin{array}{l}\text { Predict which } \\
\text { color blocks } \\
\text { fall off }\end{array}$ & Yes & $\sim 240$ \\
\hline Caramazza, McCloskey, and Green (1981) & & $\begin{array}{l}\text { Swinging pendulum } \\
\text { is cut }\end{array}$ & $\begin{array}{l}\text { Draw trajectory } \\
\text { of bob }\end{array}$ & No & 4 \\
\hline Gilden and Proffitt (1994) & & $\begin{array}{l}\text { Two circular objects } \\
\text { collide }(2 \mathrm{D})\end{array}$ & Judge mass ratio & No. & 8 \\
\hline Hegarty (1992) & & $\begin{array}{l}\text { Pulley system } \\
\text { with rope }\end{array}$ & $\begin{array}{l}\text { Verbally predict } \\
\text { how pulley rotates }\end{array}$ & Yes & $1 / 4$ \\
\hline Kubricht et al. (2016) & & $\begin{array}{l}\text { Two glasses of } \\
\text { liquid are tipped }\end{array}$ & $\begin{array}{l}\text { Predict verbally } \\
\text { which spills first }\end{array}$ & Yes & $\sim 10^{24} / 240,000^{10}$ \\
\hline Lawson (2006) & & $\begin{array}{l}\text { Swimming carrying } \\
\text { three weight belts } \\
\text { (Anecdotal) }\end{array}$ & $\begin{array}{l}\text { Bring belts, but } \\
\text { avoid actually } \\
\text { drowning }\end{array}$ & No & Many \\
\hline \multirow[t]{3}{*}{ This paper } & 1 & Towers of blocks & $\begin{array}{l}\text { Verbal identification } \\
\text { of final state }\end{array}$ & No & 120 \\
\hline & 2 & $\begin{array}{l}\text { Racing } \\
\text { Rube Goldberg machines }\end{array}$ & $\begin{array}{l}\text { Which completes } \\
\text { first? }\end{array}$ & No & 4 \\
\hline & 3 & $\begin{array}{l}\text { Two balls in flight } \\
\text { collide }\end{array}$ & $\begin{array}{l}\text { Probability of } \\
\text { specified outcome }\end{array}$ & No & 8 \\
\hline \multirow[t]{2}{*}{ Proffitt et al. (1990) } & 1 & $\begin{array}{l}\text { Two wheels of } \\
\text { different features }\end{array}$ & $\begin{array}{l}\text { Predict which } \\
\text { rolls faster }\end{array}$ & No & 2 \\
\hline & 2 & Rolling wheel & $\begin{array}{l}\text { Draw trajectory } \\
\text { of a point on } \\
\text { the wheel }\end{array}$ & No & $1 / 2$ \\
\hline Schwartz and Black (1996a) & & $\begin{array}{l}\text { Two meshed gears } \\
\text { with bump and dent }\end{array}$ & $\begin{array}{l}\text { Verbally predict } \\
\text { whether bump } \\
\text { meets dent }\end{array}$ & Yes & $1 / 2$ \\
\hline Schwartz and Black (1999) & & $\begin{array}{l}\text { Two glasses of } \\
\text { water }\end{array}$ & $\begin{array}{l}\text { Verbally predict } \\
\text { which glass must } \\
\text { be tipped further }\end{array}$ & Yes & 2 \\
\hline \multirow[t]{3}{*}{ Smith, Battaglia, and Vul (2013) } & 1 & $\begin{array}{l}\text { Swinging } \\
\text { pendulum is cut }\end{array}$ & $\begin{array}{l}\text { Predict where } \\
\text { ball lands }\end{array}$ & Yes & 4 \\
\hline & 2 & $\begin{array}{l}\text { Swinging } \\
\text { pendulum is cut }\end{array}$ & $\begin{array}{l}\text { Decide where } \\
\text { to cut string }\end{array}$ & Yes & 4 \\
\hline & 3 & $\begin{array}{l}\text { Swinging } \\
\text { pendulum is cut }\end{array}$ & $\begin{array}{l}\text { Draw trajectory } \\
\text { of bob }\end{array}$ & No & 4 \\
\hline Smith, Dechter, et al. (2013) & & $\begin{array}{l}\text { Ball bounces } \\
\text { in fixed frame }\end{array}$ & $\begin{array}{l}\text { Decide whether ball } \\
\text { reached red or } \\
\text { green first }\end{array}$ & $\begin{array}{l}\text { Mostly yes, } \\
\text { some no }\end{array}$ & 2 \\
\hline Téglás et al. (2011) & & $\begin{array}{l}\text { Objects bounce } \\
\text { in container } \\
\text { with hole }\end{array}$ & $\begin{array}{l}\text { Infants are } \\
\text { surprised by } \\
\text { improbable outcome }\end{array}$ & Yes & 16 \\
\hline
\end{tabular}

Table 3: Comparison of Tasks. DoF $=$ Degrees of freedom is a physical feature: the dimensionality of the phase space (in a natural construal) through which the system evolves. NoP $=$ Number of parameters is a computational feature; the number of real-valued parameters that a simulator would have to track for accurate prediction. Generally these are equal, but sometimes they are different. 


\subsubsection{What is the role of memory limitations in physical reasoning?}

The size of people's working memory is very limited. It seems plausible that that limitation is involved in our findings in Experiment 1; maybe people simply cannot simultaneously keep track of ten blocks in motion (Levin \& Simons, 1997; Most, Simons, Scholl, \& Chabris, 2000; Vul, Alvarez, Tenenbaum, \& Black, 2009). It might also be involved in the results of Experiment 2 - keeping track of the state of the two tracks simultaneously overburdens working memory.

One interesting hypothesis is that a systematic review of past physical reasoning tasks should show some performance degradation for cases where many objects need to be represented. Table 3 estimates the Degrees of Freedom and Number of Parameters for the studies it contains. The studies differ vastly in the complexity of the systems they ask participants to reason about. Notably, however, the complexity of the system seems to be largely unrelated to whether the results appear to be consistent or inconsistent with simulation. We actually find this result rather surprising. One possible explanation for the failure to see this degradation is that counting objects is a very rough way of estimating complexity. It is possible that people invoke simplifying representational schemes in some cases (e.g., chunking objects together, etc...).

Within this broader context, the studies in this paper are no more taxing than those which have been interpreted as being consistent with simulation. In Experiment 1, our block towers were composed of 10 blocks each, the same as in studies conducted in support of PMST (e.g. Battaglia et al., 2013). In Experiment 2, there were only a small number of dynamic objects in each scene; in two thirds of the scenes, the number of dynamic objects was exactly two. Finally, in Experiment 3 , each scene had only two dynamic objects. So while we suppose that memory limitations do play a role, they might not be the only factor explaining our results.

\subsection{Conclusions}

Simulation has been argued to be an important and effective way in which people reason about the physical world. In this paper we ask about the limits on the use of simulation as a strategy. Across three studies, we found empirical contradictions to the natural predictions made by PMST, in domains where one might expect the theory to apply. However, in all three cases, considerations from the broader literature on human cognitive and perceptual limitations suggested that these tasks might be difficult for people.

The design of our experiments tried to mimic many of the empirical studies which have supported PMST in complexity and content. Thus we believe they represent an interesting testbed for the generalization of the theory.

There is strong evidence for the use of highly isomorphic mental simulation in certain tasks, including image scanning (Kosslyn, Ball, \& Reiser, 1978), mental rotation (Shepard \& Metzler, 1988), and judgments of containment for single objects (Smith et al., 2017). There are also tasks for which the consensus is that mental simulation is not an accurate account, as in the "How far will the blocks come to rest? [sic]" task reported in the appendix to Battaglia et al. (2013). Where the dividing line stands, what features of the task or of the situation constrain the use of simulation, and what the mind does when it switches away from the use of simulation are all open questions. While we do have some relevant data, more evidence will be needed to make progress on this front.

As the field tries to grapple with these complex questions, we argue that any complete account of human physical reasoning must contend with both the cases where people appear to do well and the situations where they apparently are limited or deceived. As a result, experiments exposing the limits of simulation can be as informative as those that show the successes.

\section{Acknowledgements}

The authors would like to thank Ellie Robbins, Michael Lepori, Xuechen Sheryl Zhang, and Adi Kwiatek for help with this research, Taylor Hadden for help with the modeling, and Gregory L. Murphy, Gary F. Marcus, and Kevin A. Smith for helpful comments.

\section{References}

Barsalou, L. W., et al. (1999). Perceptual symbol systems. Behavioral and brain sciences, 22(4), $577-660$. 
Bates, C. J., Yildirim, I., Tenenbaum, J. B., \& Battaglia, P. W. (2015). Humans predict dynamics using probabilistic simulation. In Proceedings of the 37 th annual conference of the cognitive science society.

Battaglia, P. W., Hamrick, J. B., \& Tenenbaum, J. B. (2013, November). Simulation as an engine of physical scene understanding. Proceedings of the National Academy of Sciences, 110(45), $18327-18332$.

Bobrow, D. G. (1984). Qualitative reasoning about physical systems: an introduction. Artificial intelligence, $24(1-3), 1-5$.

Caramazza, A., McCloskey, M., \& Green, B. (1981). naive beliefs in "sophisticated" subjects. , 7.

Cooper, L. A. (1976). Demonstration of a mental analog of an external rotation. Perception \& Psychophysics, 19(4), 296-302.

Dasgupta, I., Smith, K. A., Schulz, E., Tenenbaum, J. B., \& Gershman, S. J. (2018). Learning to act by integrating mental simulations and physical experiments. BioRxiv, 321497.

Davis, E., \& Marcus, G. (2015, June). The scope and limits of simulation in cognitive models. arXiv preprint arXiv:1506.04956.

Davis, E., \& Marcus, G. (2016, April). The scope and limits of simulation in automated reasoning. Artificial Intelligence, 233, 60-72.

Davis, E., Marcus, G., \& Frazier-Logue, N. (2017). Commonsense reasoning about containers using radically incomplete information. Artificial intelligence, 248, 46-84.

Firestone, C., \& Scholl, B. (2016). Seeing stability: Intuitive physics automatically guides selective attention. Journal of Vision, 16(12), 689-689.

Firestone, C., \& Scholl, B. (2017). Seeing physics in the blink of an eye. Journal of Vision, 17(10), 203-203.

Forbus, K. D. (1984). Qualitative process theory. Artificial intelligence, 24(1-3), 85-168.

Forbus, K. D., \& Gentner, D. (1983). Learning physical domains: Toward. Machine Learning: An Artificial Intelligence Approach, Volume II, 2, 311.

Gentner, D. (1983). Structure-mapping: A theoretical framework for analogy. Cognitive science, 7(2), 155-170.

Gentner, D., \& Gentner, D. R. (1983). Flowing waters or teeming crowds: Mental models of electricity. Mental models, 99, 129.

Gerstenberg, T., Zhou, L., Smith, K. A., \& Tenenbaum, J. B. (2017). Faulty towers: A hypothetical simulation model of physical support. In Proceedings of the 39th annual meeting of the cognitive science society, cogsci 2017, london, uk, 16-29 july 2017.

Gigerenzer, G. (1991). How to make cognitive illusions disappear: Beyond "heuristics and biases". European Review of Social Psychology, 2(1), 83-115.

Gigerenzer, G. (1996). On narrow norms and vague heuristics: A reply to Kahneman and Tversky. Psychological Review, 103, 592-596.

Gilden, D. L., \& Proffitt, D. R. (1994). Heuristic judgment of mass ratio in two-body collisions. Perception \& Psychophysics, 56(6), 708-720.

Grice, H. P. (1991). Studies in the way of words. Harvard University Press.

Griffiths, T. L., \& Tenenbaum, J. B. (2007). Two proposals for causal grammars. Causal learning: Psychology, philosophy, and computation, 323-345.

Hamrick, J. B., Battaglia, P. W., Griffiths, T. L., \& Tenenbaum, J. B. (2016, December). Inferring mass in complex scenes by mental simulation. Cognition, 15\%, 61-76.

Hamrick, J. B., Smith, K. A., Griffiths, T. L., \& Vul, E. (2015). Think again? The amount of mental simulation tracks uncertainty in the outcome. In Proceedings of the 37th annual conference of the cognitive science society.

Hegarty, M. (1992). Mental animation: Inferring motion from static displays of mechanical systems. Journal of Experimental Psychology: Learning, Memory, and Cognition, $18(5), 1084$.

Hegarty, M. (2004a). Mechanical reasoning by mental simulation. Trends in cognitive sciences, $8(6), 280-285$.

Hegarty, M. (2004b, June). Mechanical reasoning by mental simulation. Trends in Cognitive Sciences, 8(6), 280-285.

Hertwig, R., \& Gigerenzer, G. (1999). The 'conjunction fallacy' revisited: how intelligent inferences look like reasoning errors. Journal of Behavioral Decision Making, 12(4), 31.

Holmes, K. J., \& Wolff, P. (2010). Simulation from schematics: dorsal stream processing and the perception of implied motion. In Proceedings of the annual meeting of the cognitive science society (Vol. 32). 
Johnson-Laird, P. (1998). Imagery, visualization, and thinking. Perception and cognition at century's end, 441-467.

Johnson-Laird, P. N. (1980). Mental models in cognitive science. Cognitive science, 4(1), 71-115.

Johnson-Laird, P. N. (2010). Mental models and human reasoning. Proceedings of the National Academy of Sciences, 107(43), 18243-18250.

Kaufman, E. L., Lord, M. W., Reese, T. W., \& Volkmann, J. (1949). The Discrimination of Visual Number. The American Journal of Psychology, 62(4), 498-525.

Kennedy, R., Clifford, S., Burleigh, T., Waggoner, P., \& Jewell, R. (2018). The shape of and solutions to the mturk quality crisis. Unpublished manuscript.

Klenk, M., \& Forbus, K. (2009). Analogical model formulation for transfer learning in ap physics. Artificial intelligence, 173(18), 1615-1638.

Kline, P. (2013). Handbook of psychological testing. Routledge.

Kosslyn, S. M., Ball, T. M., \& Reiser, B. J. (1978). Visual images preserve metric spatial information: evidence from studies of image scanning. Journal of experimental psychology: Human perception and performance, 4(1), 47.

Kozhevnikov, M., \& Hegarty, M. (2001). Impetus beliefs as default heuristics: Dissociation between explicit and implicit knowledge about motion. Psychonomic Bulletin \& Review, 8(3), 439453.

Kruschke, J. K. (2013). Bayesian estimation supersedes the t test. Journal of Experimental Psychology: General, 142(2), 573-603.

Kubricht, J. R., Holyoak, K. J., \& Lu, H. (2017, October). Intuitive Physics: Current Research and Controversies. Trends in Cognitive Sciences, 21(10), 749-759.

Kubricht, J. R., Jiang, C., Zhu, Y., Zhu, S.-C., Terzopoulos, D., \& Lu, H. (2016). Probabilistic simulation predicts human performance on viscous fluid-pouring problem. In Proceedings of the 38th annual conference of the cognitive science society (pp. 1805-1810).

Kubricht, J. R., Zhu, Y., Jiang, C., Terzopoulos, D., Zhu, S.-C., \& Lu, H. (2017). Consistent probabilistic simulation underlying human judgment in substance dynamics. In Proceedings of the 39th annual meeting of the cognitive science society (pp. 700-705).

Lawson, R. (2006). The science of cycology: Failures to understand how everyday objects work. Memory 8 cognition, 34 (8), 1667-1675.

LeCun, Y., Bengio, Y., \& Hinton, G. (2015). Deep learning. nature, 521(7553), 436-444.

Levin, D. T., \& Simons, D. J. (1997). Failure to detect changes to attended objects in motion pictures. Psychonomic Bulletin E Review, 4(4), 501-506.

Ludwin-Peery, E., Bramley, N., Davis, E., \& Gureckis, T. (2020). Broken physics: A conjunction fallacy effect in intuitive physical reasoning. Psychological Science, 31(12), 1602-1611.

Marcus, G. F., \& Davis, E. (2013, December). How Robust Are Probabilistic Models of HigherLevel Cognition? Psychological Science, 24(12), 2351-2360.

McCloskey, M., Caramazza, A., \& Green, B. (1980). Curvilinear motion in the absence of external forces: Naïve beliefs about the motion of objects. Science, 210(4474), 1138-1141.

Miller, G. A. (1956). The magical number seven, plus or minus two: Some limits on our capacity for processing information. Psychological review, 63(2), 81.

Most, S. B., Simons, D. J., Scholl, B. J., \& Chabris, C. F. (2000). Sustained inattentional blindness. Psyche, 6(14).

Proffitt, D. R., Kaiser, M. K., \& Whelan, S. M. (1990, July). Understanding wheel dynamics. Cognitive Psychology, 22(3), 342-373.

Pylyshyn, Z. W. (2002). Mental imagery: In search of a theory. Behavioral and brain sciences, $25(2), 157$.

Sanborn, A. N. (2014). Testing bayesian and heuristic predictions of mass judgments of colliding objects. Frontiers in psychology, 5, 938.

Sanborn, A. N., Mansinghka, V. K., \& Griffiths, T. L. (2013). Reconciling intuitive physics and newtonian mechanics for colliding objects. Psychological review, 120(2), 411.

Schwartz, D. L., \& Black, J. B. (1996a). Analog imagery in mental model reasoning: Depictive models. Cognitive Psychology, 30(2), 154-219.

Schwartz, D. L., \& Black, J. B. (1996b). Shuttling between depictive models and abstract rules: Induction and fallback. Cognitive science, 20(4), 457-497.

Schwartz, D. L., \& Black, T. (1999). Inferences through imagined actions: Knowing by simulated doing. Journal of Experimental Psychology: Learning, Memory, and Cognition, 25(1), 116.

Shepard, S., \& Metzler, D. (1988). Mental rotation: effects of dimensionality of objects and type 
of task. Journal of experimental psychology: Human perception and performance, 14(1), 3. Siegler, R. S. (1976). Three aspects of cognitive development. Cognitive psychology, 8(4), 481-520. Sloman, S. (2005). Causal models: How people think about the world and its alternatives. Oxford University Press.

Smith, K. A., Battaglia, P., \& Vul, E. (2013). Consistent physics underlying ballistic motion prediction. In Proceedings of the annual meeting of the cognitive science society (Vol. 35).

Smith, K. A., Battaglia, P. W., \& Vul, E. (2018). Different physical intuitions exist between tasks, not domains. Computational Brain \& Behavior, 1-18.

Smith, K. A., Dechter, E., Tenenbaum, J. B., \& Vul, E. (2013). Physical predictions over time. In Proceedings of the annual meeting of the cognitive science society (Vol. 35).

Smith, K. A., Peres, F., Vul, E., \& Tenebaum, J. (2017). Thinking inside the box: Motion prediction in contained spaces uses simulation. In Cogsci.

Smith, K. A., \& Tenenbaum, J. B. (2013). Physical predictions over time. In Proceedings of the annual meeting of the cognitive science society (Vol. 35).

Smith, K. A., \& Vul, E. (2013). Sources of uncertainty in intuitive physics. Topics in cognitive science, 5(1), 185-199.

Strevens, M. (2013). Tychomancy. Harvard University Press.

Téglás, E., Vul, E., Girotto, V., Gonzalez, M., Tenenbaum, J. B., \& Bonatti, L. L. (2011). Pure reasoning in 12-month-old infants as probabilistic inference. Science, 332(6033), 1054-1059.

Tversky, A., \& Kahneman, D. (1983, October). Extensional Versus Intuitive Reasoning: The Conjunction Fallacy in Probability Judgment. Psychological Review, 90(4), 23.

Ullman, T. D., Spelke, E., Battaglia, P., \& Tenenbaum, J. B. (2017, September). Mind Games: Game Engines as an Architecture for Intuitive Physics. Trends in Cognitive Sciences, 21(9), 649-665.

Ullman, T. D., Stuhlmüller, A., Goodman, N. D., \& Tenenbaum, J. B. (2018). Learning physical parameters from dynamic scenes. Cognitive psychology, 104, 57-82.

Unity. (2005). Retrieved 2018-06-20, from https://unity3d.com

Vul, E., Alvarez, G., Tenenbaum, J., \& Black, M. (2009). Explaining human multiple object tracking as resource-constrained approximate inference in a dynamic probabilistic model. Advances in neural information processing systems, 22, 1955-1963.

Wang, Z., Bovik, A. C., Sheikh, H. R., \& Simoncelli, E. P. (2004). Image quality assessment: from error visibility to structural similarity. IEEE transactions on image processing, 13(4), $600-612$.

Wickham, H. (2016). ggplot2: Elegant graphics for data analysis. Springer-Verlag New York. Retrieved from http://ggplot2.org

Wolff, P. (2007). Representing causation. Journal of experimental psychology: General, 136(1), 82.

Wolff, P., \& Barbey, A. K. (2015). Causal reasoning with forces. Frontiers in human neuroscience, $9,1$. 


\section{A Appendix 1}

Table 4: Experiment 1: Edit Distance Heuristic Scores

\begin{tabular}{lrrrr}
\hline Trial Type & Actual & Foil 1 & Foil 2 & Foil 3 \\
\hline 1. Reshape 1 block & 0 & -1 & -1 & -1 \\
2. Reshape 1 block & 0 & -1 & -1 & -1 \\
3. Recolor 1 block & 0 & -1 & -1 & -1 \\
4. Recolor 1 block & 0 & -1 & -1 & -1 \\
5. Reverse colors & 0 & 0 & 0 & 0 \\
6. Reverse colors & 0 & -2 & -2 & -2 \\
7. Plus 1 block & 0 & -2 & -2 & -2 \\
8. Plus 1 block & 0 & -2 & -2 & -2 \\
9. Minus 1 block & 0 & -2 & -2 & -2 \\
10. Minus 1 block & 0 & -2 & -2 & -2 \\
11. Minus 2 blocks & 0 & -4 & -4 & -4 \\
12. Minus 2 blocks & 0 & -4 & -4 & -4 \\
13. Minus 3 blocks & 0 & -6 & -6 & -6 \\
14. Minus 3 blocks & 0 & -6 & -6 & -6 \\
15. Multiple changes & 0 & -2 & -2 & -2 \\
16. Multiple changes & 0 & -10 & -8 & -5 \\
17. Multiple changes & 0 & -1 & -9 & -10 \\
18. Multiple changes & 0 & -6 & -6 & -6 \\
19. Multiple changes & 0 & -11 & -5 & -8 \\
20. Multiple changes & 0 & -7 & -4 & -9 \\
21. Multiple changes & 0 & -10 & -6 & -7 \\
22. Multiple changes & 0 & -10 & -6 & -10 \\
23. Multiple changes & 0 & -5 & -9 & -7 \\
24. Multiple changes & 0 & -3 & -4 & -6 \\
25. Noise only & 0 & 0 & 0 & 0 \\
26. Noise only & 0 & 0 & 0 & 0 \\
27. Noise only & 0 & 0 & 0 & 0 \\
28. Noise only & 0 & 0 & 0 & 0 \\
\hline
\end{tabular}

